July 24, 2001

\title{
A Search Theory of Money and Commerce with Neoclassical Production
}

\author{
Miquel Faig* \\ Department of Economics \\ University of Toronto \\ 150 St.George Street \\ Toronto, Canada M5S 3G7 \\ www.economics.utoronto.ca/faig/ \\ E-mail: faig@chass.utoronto.ca
}

\begin{abstract}
This paper advances a highly tractable model with search theoretic foundations for money and neoclassical growth. In the model, manufacturing and commerce are distinct and separate activities. In manufacturing, goods are efficiently produced combining capital and labor. In commerce, goods are exchanged in bilateral meetings. The model is applied to study the effects of inflation on capital accumulation and welfare. With realistic parameters, inflation has large negative effects on welfare even though it raises capital and output. In contrast, with cash-in-advance, a device informally motivated with bilateral trading, inflation depresses capital and output and has a negligible effect on welfare.
\end{abstract}

Keywords: search, money, commerce, inflation, neoclassical production, capital accumulation, optimum quantity of money.

JEL Classicification codes: E40, E52, E13.

*This paper was elaborated while visiting the Universitat Pompeu Fabra in Barcelona. I benefited from comments made at the seminars in the University Pompeu Fabra and the University Autonoma of Barcelona. 


\section{Introduction}

This paper advances a model to bring together the search theoretic foundations for money and the neoclassical growth model. The objective of the paper is to contribute to a reunification of monetary economics, which is presently divided into two separate subfields. One of these subfields studies the foundation for the existence of money through the endogenous determination of trading patterns and the media of exchange. In this subfield the search theoretic paradigm has been dominant after the work of Kiyotaki and Wright (1989). Unfortunately, in this paradigm it has been difficult to deal with many practical problems such as the interaction between money and capital accumulation. For this reason, a separate subfield of monetary economics studies these practical problems in models that combine the neoclassical growth framework with an ad-hoc demand for money. In these models, money is typically wanted because either it is an argument of the utility function, it is an input in a transactions technology, or buyers must satisfy a cash-in-advance constraint. The purpose of this work is to advance towards a comprehensive theory of money with a model that combines the virtues of the two subfields of monetary economics. That is, I advance a model that provides the foundations for money of the search paradigm in a tractable neoclassical growth framework.

The most distinctive feature of the model I advance is a realistic separation between manufacturing and commerce as two different sectors. In the manufacturing sector, capital and labor is efficiently combined as in the neoclassical growth model, but production takes one period to be completed. At the beginning of each period, the predetermined stock of goods is divided into two parts. One part is used as capital in manufacturing. The other part is used for trading in commerce. In the commercial sector, traders are paired bilaterally as in other monetary search models. Moreover, traders are anonymous, so all trades must be not only mutually advantageous but also quid pro quo.

The fact that production is done prior to exchange allows for an endogenous determination of the media of exchange as in the earlier models of Kiyotaki and Wright (1989 and 1993). This endogenous determination of the media of exchange faded away in the search theoretic literature when, to endogenize prices, production was assumed to be instantaneous with consumption (see Shi (1995) and Trejos and Wright(1995)). The present model in the complete form of Section 3 combines both endogenous prices and endogeneous media of exchange.

To be able to insert a commercial sector with bilateral matching in a neoclassical growth frame- 
work, my model incorporates recent breakthroughs in the search theoretical foundations for money. Following Shi (1997), households are composed of a large number of individuals who share all their capital, their money balances, and their consumption. This allows perfect risk sharing inside the household, so it obviates the difficult problem of a non-degenerate distribution of money and capital. ${ }^{1}$ Following Laing, Li, and Wang (2000), the size of transactions is endogenously determined by the balance between the desire for a diverse basket of goods and the cost of having to perform more transactions when the number of goods consumed increases. Finally, price setting by sellers is compatible with a monetary equilibrium because, as in Soller-Curtis and Wright (2000), sellers face potential buyers with unknown preferences. In the present set up, the most preferred set of features wanted in a consumption good differs continuously across households. Sellers must make offers without knowing how much the buyers they are facing like the particular merchandise they sell. Consequently, sellers cannot extract the whole trading surplus despite being able to make take-it-or-leave-it offers to buyers. $^{2}$

Bilateral matching in the commercial sector provides the foundation for the existence of money. However, the role of bilateral matching in the present model goes well beyond a motivation for a cash-in-advance constraint or a transactions technology. For example, with a simple cash-in-advance constraint the velocity of money remains unchanged when the opportunity cost of holding money increases, let us say due to higher inflation. With a transactions technology, velocity increases with higher inflation because households spend more time performing transactions to economize in money balances. In contrast, in the present model the velocity of money increases with higher inflation for a completely different reason. Households respond to higher inflation not by spending more time performing transactions but by spending less time shopping. When money is more costly to hold, households reduce the number of purchases they make, buying in each purchase a larger quantity. Therefore, higher inflation induces a sacrifice in the diversity of goods consumed. In the extreme case of a hyperinflation, the model predicts, quite realistically, that people buy the goods they encounter in large quantities without much regard for a balanced shopping basket.

Another major difference between the present model and those with a cash-in-advance con-

\footnotetext{
${ }^{1}$ See Molico (1998) for a search model that incorporates divisible money and goods without the representative household device. Also, see Taber and Wallace (1999) and Zhou (1999) for search models where individuals hold non-degenerate inventories of indivisible money.

${ }^{2}$ Convexity in preferences of potential buyers eliminates the "law of two prices" of Soller-Curtis and Wright (2000) for an analogous reason to the one that eliminates the "law of two techniques" in production economics in the presence of convex isoquants.
} 
straint is found in the welfare properties of an equilibrium. With a cash-in-advance constraint an equilibrium path is efficient if the opportunity cost of holding money is brought to zero as in the optimum quantity of money rule defended by Friedman (1969). In the present model, even though it is optimal to follow Friedman's rule under reasonable conditions, the economy remains inefficient when this rule is followed. Efficiency requires that buyers pay the marginal cost of producing a commodity. In principle, this could be achieved if buyers had the bargaining power to make take-it-or-leave-it offers to sellers. However, this would be inconsistent with the existence of a commercial sector. If buyers were to make take-it-or-leave-it offers, they would extract the whole trading surplus, so sellers would disappear from the market. For sellers to exist, they must be able to cover not only the cost of producing what they sell but also the cost of servicing all sales, successful or not, and the cost of waiting for customers. These fixed costs of commerce must be covered with a markup of price over cost. ${ }^{3}$

Money in my model is neutral but not superneutral. An increase in the rate of monetary growth increases inflation and as a result the ratio of buyers over sellers falls. As a consequence, traders meet less efficiently in the commercial sector, so there is a shift of labor from this sector to the production of goods and home services. The end result of higher inflation is the production of more output and the accumulation of more capital. Despite this affluence of goods, welfare, under reasonable conditions, declines with inflation. Inflation increases measured consumption, but it reduces the diversity of the goods consumed. For realistic parameters, the welfare costs of inflation are large, sufficiently so to rationalize a return to gold or "dollarization" when countries are unable to control even moderate annual rates of inflation with their national currency. In contrast, the welfare cost of moderate inflation in models with a cash-in-advance constraint is negligible. Also, in these models inflation raises output and capital.

The present model is related to recent contributions by Shi (1999 and 2001) who also add capital to search monetary models with a large representative household. My model differs from these earlier contributions in many respects, all of them interrelated. Contrary to Shi's contributions, my model has the following characteristics. Manufacturing and commerce are two separate activities, instead of being two facets of the same activity. Capital is a perfect substitute for output, instead of being a perfect substitute for consumption. Sellers are the traders that set prices, instead of those

\footnotetext{
${ }^{3}$ The elimination of the commercial mark-up could be achieved with lump-sum fees that buyers would pay for the right of shopping with a seller. These fees are found in some discount stores. These fees are so rare probably because they reduce the sellers' incentive to provide good merchandise and good service.
} 
being set by buyers or by a bargaining game. The endogenous size of transactions is determined by a desire for variety in the basket of goods, instead of being determined by decreasing returns in production. Money is specialized in the purchase of consumption goods, excluding capital. And both the opportunity cost of labor and the allocation of individuals inside the household are completely endogenous.

The study of the effects of inflation has several precedents in search theoretical models. Li $(1995)^{4}$ and Laing, Li, and Wang (2000) stress that an increase in inflation increases search effort to speed up the velocity of money. Shi $(1997,1998,1999$, and 2001) stresses that in search models inflation not only induces households to find ways to reduce their money balances, but also changes the composition of market participants and hence trading opportunities. In all these earlier contributions, inflation potentially increases output and welfare. In the present paper, inflation may also do both: speed up search and change the composition of market participants. However, the mechanisms for these effects are very different from earlier contributions. For example, inflation may speed up search not by increasing search effort but by making buyers less picky about the goods' varieties they buy. ${ }^{5}$ Also, inflation no longer improves production efficiency as in Shi's papers. Instead, inflation just worsens trade efficiency.

In summary, the main conclusions of the paper are the following. It is possible to reunite monetary theory with a model that combines the foundations for money of the search paradigm with the practical uses opened by the neoclassical growth framework. In the model, bilateral matching not only motivates the existence of money and commerce, but it also induces effects quite different from cash-in-advance or a transactions technology. Despite the many elements of the model, it is quite manageable, so it can be used for the analysis of many issues in monetary economics. In the paper, the model is applied to study the effects of inflation on capital accumulation and welfare. Under reasonable conditions, inflation depresses welfare even though it raises output and capital. Also, with reasonable parameters the costs of inflation are much larger than those found in models with a cash-in-advance constraint. In fact, the model can provide a rationale to "dollarization" in countries that are unable to control even moderate inflations with their national currency.

The paper is organized as follows. Section 2 analyzes a simple version of the model where goods are perishable if they are not invested as capital. This assumption precludes commodity money, so only fiat money can play the role of medium of exchange. This section shows that capital

\footnotetext{
${ }^{4} \mathrm{Li}$ (1995) approximates the effects of inflation with a direct tax on money.

${ }^{5}$ This effect cancels when buyers have no bargaining power, but it is found in the model sketched at the end of Subsection 4.4.
} 
accumulation takes place according to the same dynamics as in the neoclassical growth model and discusses properties of the demand for money. Section 3 allows for goods to be durable even when

they are not employed as capital. It further assumes that if a good, let us say a piece of gold, is used as a medium of exchange, the same piece of gold cannot be simultaneously used as an input in manufacturing. With this assumption this section describes equilibria with commodity money. Also, it discusses the conditions for the existence of valuable fiat money. Section 4 analyzes the effects of inflation on capital accumulation and welfare and describes the welfare properties of an equilibrium. Finally, Section 5 concludes.

\section{The Basic Model}

The economy is populated by a $[0,1]$ continuum of infinitely lived households who produce and consume differentiated goods. Households do not consume the goods they produce so they need to trade. In addition to consuming goods, households enjoy home services, or equivalently leisure activities, which can neither be traded nor stored. Consumption of both goods and home services is shared equally by all members of the household. The exchange of goods is performed in decentralized markets. All trades in the market are bilateral and must be mutually beneficial to both parties. Market participants are anonymous so exchanges must be quid pro quo.

Goods are perfectly divisible. Once produced, goods either have to be consumed in the same period or have to be invested as productive capital by the same household who has produced them. This assumption is convenient to study equilibria with fiat money because it precludes the use of goods as media of exchange. However, as seen in the next section, the basic logic of the model does not depend on it.

There are $H$ types of households and $H$ types of goods (indexed both by $h \in H$ ), where $H \geq 3$. Each good $h$ comes in a continuum of varieties (indexed by $i \in[0,1]$ ) distributed around a unit circle. Household $h i$ produces good $h$ variety $i$ and can consume all varieties of good $h+1$ modulus $H$. However, it does not like all these varieties the same. For concreteness, let us say that household $h i$ likes variety $i$ of good $h+1$ the best. The utility of the other varieties declines the further apart they are from $i$. Households are evenly spread over the set of goods and varieties they produce.

Since $H \geq 3$, two households are unable to mutually satisfy their consumption needs in a barter exchange. The absence of barter combined with the impossibility of goods being used as media of exchange implies that all exchange in this economy must use money. Money consists of storable 
objects, referred to as dollars, that are useless for consumption or production. These objects are perfectly divisible and can be created without cost by the government, who has the monopoly to do so.

\subsection{Households' Decisions}

Without loss of generality, I describe the actions of household $h 0$. In this description, I adopt the following notation. Lower-case letters denote household's $h 0$ decision variables. Upper-case variables denote the decisions of other households, which are taken as given by household $h 0$. In a symmetric equilibrium, lower-case letters are equal to the corresponding upper-case letters.

Each household is composed of a large countable number of individuals $J$. Time is discrete. In each period, the individuals of a household are assigned to one of four different tasks: production of market goods (producers), production of home services (home workers), purchase of commodities (buyers), and sale of market goods (sellers). No individual can perform more than one of these tasks in the same period. Say that the production of market goods, the production of home services, and exchange are performed in different venues. Moreover, each one of the $H$ goods has a different market place.

A typical day in the life of household $h 0$ proceeds as follows. In the morning of day $t$, the household starts with a given stock of good $h 0$ to be denoted $a_{t}$. This stock is divided in two piles. One pile is destined to be the capital stock $k_{t}$ useful for production of market goods. The other pile $v_{t}$ is transferred to a warehouse of the commercial sector that the household's sellers have access. The $J$ individuals of the household are then divided into producers $n_{t} J$, home workers $l_{t} J$, buyers $b_{t} J$, and sellers $s_{t} J$. Sellers go to the market for good $h$ and if they meet a buyer they announce a take-it-or-leave-it offer. These offers specify the quantity of good supplied $q_{t}$ and the payment in money demanded $z_{t}$ of a transaction (the output price is $p_{t}=z_{t} / q_{t}$ ). Offers have to be made without knowledge of the most preferred variety of the buyer faced in a meeting. ${ }^{6}$ Buyers get an equal share of the money of the household and travel to the market for good $h+1$. Upon meeting

\footnotetext{
${ }^{6}$ The trade meeting is assumed to proceed in such a way that the preferred variety of the buyer is not revealed. For example, this is the outcome if the buyer moves first with an action that consists in either showing an unequivocal mark that reveal his type or declining to do so. Next, the seller makes an offer. Finally, the buyer decides accepting the offer or not. Given this sequence of events, the seller appropriates the whole trade surplus if he infers the type of the buyer. Consequently, the buyer has no incentive to reveal his type. For this result, it is crucial that the seller cannot commit to a payment schedule and communicate it to the buyer prior his decision to reveal the type. The richer model where this is possible is much more complicated and will be dealt in future research.
} 
a seller, they observe the variety for sale, receive an offer $\left(Q_{t}, Z_{t}\right)$, and decide if they accept the offer or not. The producers of market goods use the capital stock $k_{t}$ to generate the output that is going to be available the next day. Home workers perform the home services without any need of capital. ${ }^{7}$ In the evening, all the individuals of the household get together, and they equally share the consumption of market goods purchased and home services produced during the day.

Buyers and sellers are matched according to a matching technology specified by the two functions $\mathcal{B}$ and $\mathcal{S}$. The function $\mathcal{B}: \Re_{+}^{3} \rightarrow[0,1]$ maps $b_{t}, B_{t}$, and $S_{t}$ onto the fraction of buyers in the household paired with a seller of good $h+1$. This seller carries with equal probability any one of the varieties of good $h+1$. Analogously, the function $\mathcal{S}: \Re_{+}^{3} \rightarrow[0,1]$ maps $s_{t}, B_{t}$, and $S_{t}$ onto the fraction of sellers in the household paired with a buyer of good $h$. This buyer prefers with equal probability any one of the varieties of good $h$. For concreteness, I assume that all individuals in a market are randomly paired once and only once each day. Also, I assume that there is an infinite number of individuals in the household $(J=\infty)$, so the law of large numbers applies. Hence,

$$
\mathcal{B}\left(b_{t}, B_{t}, S_{t}\right)=b_{t} \frac{S_{t}}{B_{t}+S_{t}} \text {, and } \mathcal{S}\left(s_{t}, B_{t}, S_{t}\right)=s_{t} \frac{B_{t}}{B_{t}+S_{t}}
$$

Most results that follow do not depend on these specific functional forms.

The objective of the household is to maximize the utility from the consumption of goods and home services. This utility is additively separable with discount factor $\beta$ :

$$
\sum_{t=0}^{\infty} \beta^{t}\left[\mathcal{U}\left(c_{t}\right)+\mathcal{V}\left(l_{t}\right)\right], \quad 0<\beta<1 .
$$

The function $\mathcal{U}: \Re_{+} \rightarrow \Re$ is logarithmic, unless otherwise specified. The function $\mathcal{V}: \Re_{+} \rightarrow \Re$ maps the fraction of home workers in the household onto the utility from the services they produce. ${ }^{8}$ This function is increasing, concave, differentiable, and $\mathcal{V}^{\prime}(0)=\infty$. The variable $c_{t}$ is a hedonic measure of consumption. This measure depends on the quantities acquired $q_{j t}$ by each individual $j$ of the household, the variety acquired in each purchase $i_{j t}$, and the number of individuals in the household $J$ :

$$
c_{t}=\left[\sum_{j=1}^{J} \frac{q_{j t}^{1-\sigma}\left(1-i_{j t}\right)}{J}\right]^{\frac{1}{1-\sigma}}, \quad \sigma \in(0,1) .
$$

\footnotetext{
${ }^{7}$ This absence of capital in home production can be easy relaxed.

${ }^{8}$ The variable $l_{t}$ can also be interepreted as leisure. Empirically, though, movements in and out of the labor force are the major contributor to the labor supply elasticity. These movements are seldom associated with major changes of leisure. Instead, they are associated with shifts such a moving child care from home to a day care. These two interpretations of $l_{t}$ differ when the model is calibrated.
} 
The parameter $\sigma$ measures the preference for diversity. When $\sigma \rightarrow 0$, the preference for diversity vanishes. The household gets maximum utility when consuming the most preferred variety $i_{j t}=0$. This utility declines linearly to 0 as $i_{j t}$ increases to 1 . The total number of potential purchases is the number of buyers that meet a seller. Each one of these buyers either accepts the offer made by the seller they meet and acquire a quantity $Q_{t}$, or reject the offer and acquire a quantity 0. Therefore, reorganizing the $j$ labels, we obtain:

$$
c_{t}=\left[\sum_{j=1}^{\mathcal{B}\left(b_{t}, B_{t}, S_{t}\right) J} \frac{Q_{t}^{1-\sigma} \mathcal{I}(j \text { accepts the offer })\left(1-i_{j t}\right)}{J}\right]^{\frac{1}{1-\sigma}},
$$

where $\mathcal{I}$ is the indicator function. Because buyers and sellers are randomly matched, $i_{j t}$ is the realization of a random variable uniformly distributed from 0 to 1 . Because of symmetry, the optimal strategy of household $h 0$ is to accept offers of varieties that satisfy $i_{j t} \leq x_{t}$ for a reservation distance $x_{t}$ and to reject other offers. Consequently, when $J \rightarrow \infty$ we can apply the law of large numbers to obtain:

$$
c_{t}=\left[Q_{t}^{1-\sigma} \mathcal{B}\left(b_{t}, B_{t}, S_{t}\right) \int_{0}^{x_{t}}(1-i) d i\right]^{\frac{1}{1-\sigma}}=Q_{t}\left[\mathcal{B}\left(b_{t}, B_{t}, S_{t}\right) x_{t}\left(1-\frac{x_{t}}{2}\right)\right]^{\frac{1}{1-\sigma}}
$$

The stock of goods available at the beginning of period $t$ is equal to the capital surviving from the previous period plus the newly obtained production:

$$
a_{t}=k_{t-1}(1-\delta)+y_{t}, \quad \delta \in(0,1)
$$

where $\delta$ is the depreciation rate. Production depends on the capital and labor employed in the previous period:

$$
y_{t}=\mathcal{F}\left(k_{t-1}, n_{t-1}\right) \text {. }
$$

The function $\mathcal{F}: \Re_{+}^{2} \rightarrow \Re_{+}$maps capital and labor used in period $t-1$ onto the output obtained in period $t$. This function is assumed continuously differentiable, increasing in both arguments, concave, and homogeneous of degree one. Also, the Inada conditions for an interior solution are assumed to apply.

The stock of goods available at the beginning of the period is divided into capital $k_{t}$ and goods for sale $v_{t}$ :

$$
a_{t}=k_{t}+v_{t}
$$

Because there is no aggregate uncertainty in a household and all sellers share their stock of goods for sale, the whole stock $v_{t}$ is sold during period $t$, so $v_{t}$ can be referred as sales (or also consumption 
expenditures). Sales in a period are equal to the buyers contacted by the sellers of a household times the fraction of buyers accepting the offer $\left(q_{t}, z_{t}\right)$ and times the quantity of goods sold in a transaction.

$$
v_{t}=\mathcal{S}\left(s_{t}, B_{t}, S_{t}\right) \mathcal{X}\left(q_{t}, z_{t}, t\right) q_{t}
$$

The function $\mathcal{X}: \Re_{+}^{3} \rightarrow[0,1]$ maps the vector $\left(q_{t}, z_{t}, t\right)$ onto the fraction of buyers in the market of good $h$ that accept purchasing $q_{t}$ units of the good for $z_{t}$ dollars in period $t$. The function $\mathcal{X}$ is assumed to be increasing in $q_{t}$, decreasing in $z_{t}$, and continuously differentiable with respect to $q_{t}$ and $z_{t}$. These assumptions are validated in an equilibrium.

The household must satisfy the following monetary budget constraint:

$$
m_{t+1}=m_{t}+T_{t}+z_{t} \mathcal{X}\left(q_{t}, z_{t}, t\right) \mathcal{S}\left(s_{t}, B_{t}, S_{t}\right)-Z_{t} x_{t} \mathcal{B}\left(b_{t}, B_{t}, S_{t}\right)
$$

The money holdings at the beginning of $t+1, m_{t+1}$, are equal to the money holdings at the beginning of $t, m_{t}$, plus the monetary lump-sum transfer received at the beginning of $t, T_{t}$, plus the revenue from sales minus the money spend in purchases. Money balances can only be positive: $m_{t+1} \geq 0$.

The fractions of individuals allocated to the four different activities in the household must add up to one:

$$
b_{t}+s_{t}+n_{t}+l_{t}=1 \text {. }
$$

All fractions of individuals must be non-negative. Moreover, the fraction of buyers is limited by the money available at the beginning of the period:

$$
b_{t} Z_{t} \leq m_{t}
$$

The household $h 0$ maximizes (2) subject to constraints (6) to (12). Constraint (11) can be eliminated by substitution of $1-b_{t}-s_{t}-n_{t}$ for $l_{t}$ into the other constraints. Likewise, expressions (6), (7), and (9) can be substituted into (8) to form a single goods' resource constraint. Therefore, we are left with the three constraints (8), (10), and (12) apart from the non-negativity of all variables. Using Lagrange multipliers $\lambda_{t} \beta^{t}, \mu_{t} \beta^{t}$, and $\nu_{t} \beta^{t}$ for these three constraints, the first order conditions for an optimum when the non-negativity constraints are not binding are:

$$
\begin{gathered}
\mathcal{U}^{\prime}\left(c_{t}\right) c_{t}^{\sigma} \frac{Q_{t}^{1-\sigma}}{1-\sigma}\left(1-x_{t}\right)=\lambda_{t} Z_{t} \\
\mu_{t}\left[\mathcal{X}\left(q_{t}, z_{t}, t\right)+q_{t} \mathcal{X}_{q}\left(q_{t}, z_{t}, t\right)\right]=\lambda_{t} z_{t} \mathcal{X}_{q}\left(q_{t}, z_{t}, t\right), \\
\mu_{t} q_{t} \mathcal{X}_{z}\left(q_{t}, z_{t}, t\right)=\lambda_{t}\left[\mathcal{X}\left(q_{t}, z_{t}, t\right)+z_{t} \mathcal{X}_{z}\left(q_{t}, z_{t}, t\right)\right]
\end{gathered}
$$




$$
\begin{gathered}
\left(\lambda_{t} z_{t}-\mu_{t} q_{t}\right) \mathcal{X}\left(q_{t}, z_{t}, t\right) \mathcal{S}_{1}\left(s_{t}, B_{t}, S_{t}\right)=\mathcal{V}^{\prime}\left(l_{t}\right), \\
\mathcal{U}^{\prime}\left(c_{t}\right) c^{\sigma} \frac{Q_{t}^{1-\sigma}}{1-\sigma}\left(x_{t}-\frac{x_{t}^{2}}{2}\right) \mathcal{B}_{1}\left(b_{t}, B_{t}, S_{t}\right)=\lambda_{t} Z_{t} x_{t} \mathcal{B}_{1}\left(b_{t}, B_{t}, S_{t}\right)+\nu_{t} Z_{t}+\mathcal{V}^{\prime}\left(l_{t}\right), \\
\mu_{t+1} \beta \mathcal{F}_{n}\left(k_{t}, n_{t}\right)=\mathcal{V}^{\prime}\left(l_{t}\right), \\
\mu_{t+1} \beta\left[1-\delta+\mathcal{F}_{k}\left(k_{t}, n_{t}\right)\right]=\mu_{t}, \\
\lambda_{t-1}=\left(\nu_{t}+\lambda_{t}\right) \beta \text { and } \nu_{t}\left(m_{t}-b_{t} Z_{t}\right)=0, \text { and } \\
\lim _{t \rightarrow \infty} \beta^{-t} \mu_{t} k_{t}=0 .
\end{gathered}
$$

Condition (13) equates the utility and the cost of accepting an offer when the variety of the good for sale is at the reservation distance $x_{t}$ from the variety the household likes most. Conditions (14) and (15) equate the marginal costs of increasing $q_{t}$ and $z_{t}$ to the marginal revenues these increases generate. Condition (16) equates the surplus generated by a seller to the cost of the seller's labor, which is equal to the marginal utility of home services. Condition (17) equates the utility of the purchases made by the marginal buyer to the value of the money spent plus the opportunity cost of holding the money carried and plus the opportunity cost of the time employed. Condition (18) equates the value of the marginal productivity of labor in the two production activities. Condition (19) equates the discounted value of the marginal productivity of capital to the value of a good today. Condition (20) equates the cost of acquiring one dollar yesterday with the discounted benefits this dollar brings today not only for its purchasing power but also for allowing extra buyers into the market. Finally (21) is a standard transversality condition.

\subsection{Equilibrium}

Definition: The set $\left\{x_{t}, q_{t}, a_{t}, k_{t}, y_{t}, v_{t}, b_{t}, s_{t}, n_{t}, l_{t}, m_{t}, z_{t}\right\}_{t=0}^{\infty}$ is a diversified symmetric monetary equilibrium (equilibrium for short) if

1. These paths solve the household optimization problem. That is, they maximize (2) subject to all choice variables being non-negative and the constraints (6) to (12) being satisfied for a given $a_{0}$ and given paths for the set of variables $\left\{Q_{t}, Z_{t}, B_{t}, S_{t} \text {, and } T_{t}\right\}_{t=0}^{\infty}$.

2. Sellers have rational expectations about the acceptability of their offers: The function $\mathcal{X}\left(q_{t}, z_{t}, t\right)$ is consistent with the choice of $x_{t}$ by households.

3. Aggregate variables are consistent with individual optimization: $Q_{t}=q_{t}, Z_{t}=z_{t}, B_{t}=b_{t}$, and $S_{t}=s_{t}$. 
4. The fractions $n_{t}, l_{t}, s_{t}$, and $b_{t}$ are all positive (diversification inside a household applies).

5. Money has value: $q_{t}>0$.

As is common in search monetary models, if $\mathcal{U}$ instead of being logarithmic has the property that $\mathcal{U}(0)$ is finite, there is a trivial non-monetary equilibrium where 1 to 3 in the previous definition hold but not 4 and 5 . That is, if individuals believe that money will have no value next period, the solution to the household's optimization problem is to revert to autarchy with $x_{t}=q_{t}=v_{t}=$ $b_{t}=s_{t}=n_{t}=k_{t}=y_{t}=0$, and $l_{t}=1$. For a combination of interest and tractability, this section focuses on equilibria where money has value and risk is diversified at the household level, so 4 and 5 hold.

In such equilibria, the first order interior conditions (13) to (21) apply. These conditions together with the constraints (6) to (12) and symmetry define a system of difference equations that determines the equilibrium path. The complexity of this system depends on monetary policy. When the government adjusts the transfer $T_{t}$ to achieve a constant and positive opportunity cost of holding money, this system simplifies to two difference equations with similar dynamics to those of the neoclassical model.

The first step to solve for an equilibrium is to find the function $\mathcal{X}$ and its derivatives with respect to $q_{t}$ and $z_{t}$. Using (13),

$$
x_{t}=1-(1-\sigma) \lambda_{t} c_{t}^{1-\sigma} Q_{t}^{\sigma-1} Z_{t} .
$$

Therefore,

$$
\mathcal{X}\left(q_{t}, z_{t}, t\right)=1-(1-\sigma) \Lambda_{t} C_{t}^{1-\sigma} q_{t}^{\sigma-1} z_{t}
$$

This implies that the derivatives of $\mathcal{X}$ obey: $q_{t} \mathcal{X}_{q}\left(q_{t}, z_{t}, t\right)=(1-\sigma)\left[1-\mathcal{X}\left(q_{t}, z_{t}, t\right)\right]$ and $z_{t} \mathcal{X}_{z}\left(q_{t}, z_{t}, t\right)=$ $-\left[1-\mathcal{X}\left(q_{t}, z_{t}, t\right)\right]$. Substituting these expressions in (14) and (15), and using that in a equilibrium $\mathcal{X}\left(q_{t}, z_{t}, t\right)=x_{t}$, we obtain:

$$
\begin{gathered}
x_{t}=\frac{\sigma}{1+\sigma} \text { and } \\
\lambda_{t} z_{t}=(1-\sigma)^{-1} \mu_{t} q_{t} .
\end{gathered}
$$

The endogenous fraction of purchased goods is constant in equilibrium. Moreover, there is a constant proportional markup, $(1-\sigma)^{-1}$, between the production value of the goods exchanged in one transaction, $\mu_{t} q_{t}$, and the value to a buyer of these goods, $\lambda_{t} z_{t}{ }^{9}$

\footnotetext{
${ }^{9}$ With the functional form of $\mathcal{X}$ in $(23)$, it is easy to check that the second order conditions for the households' optimization problem are satisfied.
} 
Define the opportunity cost of holding money as $R_{t}=\left[1-\delta+\mathcal{F}_{k}\left(k_{t-1}, n_{t-1}\right)\right]\left(1+\pi_{t}\right)-1$, where $\pi_{t}=p_{t} / p_{t-1}-1$. Using conditions (19), (20), and (25), this opportunity cost obeys:

$$
R_{t}=\frac{\lambda_{t-1}}{\lambda_{t} \beta}-1=\frac{\nu_{t}}{\lambda_{t}}
$$

Therefore, as long as $R_{t}$ is positive, condition (12) holds with equality. Using (1), (13), (16), and (24) to (26), the first order condition (17) in a symmetric equilibrium simplifies to:

$$
\frac{\sigma^{2}}{2(1+\sigma)} \frac{s_{t}}{b_{t}+s_{t}}=\frac{\sigma^{2}}{1+\sigma} \frac{b_{t}}{b_{t}+s_{t}}+R_{t} .
$$

Hence,

$$
\frac{s_{t}}{b_{t}}=\frac{\frac{\sigma^{2}}{1+\sigma}+R_{t}}{\frac{\sigma^{2}}{2(1+\sigma)}-R_{t}} \equiv \varphi_{t}\left(R_{t}\right), \quad \varphi^{\prime}>0 .
$$

The equilibrium ratio $s_{t} / b_{t}$, to be denoted $\varphi_{t}$, varies only with $R_{t}$. An increase in $R_{t}$ reduces the return of being a buyer, so the ratio of buyers to sellers drops, or equivalently $\varphi_{t}$ increases. Using (24) to (26), we can combine and simplify the first order conditions (13), and (16) to (19), to obtain the following system of equations: ${ }^{10}$

$$
\begin{gathered}
\mathcal{V}^{\prime}\left(l_{t}\right) s_{t}=\frac{2}{2+\sigma} \frac{\sigma}{1-\sigma}, \\
\mathcal{V}^{\prime}\left(l_{t}\right)=\frac{\mathcal{F}_{n}\left(k_{t}, n_{t}\right)}{1-\delta+\mathcal{F}_{k}\left(k_{t}, n_{t}\right)} \mu_{t}, \text { and } \\
q_{t}=\frac{2}{2+\sigma} \frac{1+\sigma}{\sigma}\left(\frac{b_{t}+s_{t}}{b_{t} s_{t} \mu_{t}}\right) .
\end{gathered}
$$

Equations (29) and (30) together with (28) and the resource constraints (8) and (11) determine the equilibrium values for $b_{t}, s_{t}, l_{t}, n_{t}$, and $k_{t}$ as a function of $\mu_{t}, R_{t}$, and $a_{t}$. Using these values, equilibrium $q_{t}$ is determined by (31). Finally, equilibrium $v_{t}$ is determined simplifying its definition (9) with the help of (1), (24), (31), and symmetry:

$$
v_{t}=\frac{2}{2+\sigma} \mu_{t}^{-1}
$$

To complete the dynamic system that determines the equilibrium path, we need the laws of motion for $R_{t}, \mu_{t}$, and $a_{t}$. I assume $R_{t}$ to be the target of monetary policy, which manipulates $T_{t}$ to achieve a predetermined path for $R_{t}$. The laws of motion for $\mu_{t}$ and $a_{t}$ are determined by (19), and (6) together with (7):

$$
\mu_{t+1}=\mu_{t}\left[1-\delta+\mathcal{F}_{k}\left(k_{t}, n_{t}\right)\right]^{-1} \beta^{-1} \text { and }
$$

\footnotetext{
${ }^{10}$ Equation (29) is obtained combining (5), (13), (16), and (25), and using symmetry, (1), and (24) to simplify the resulting expression. Equation (30) is obtained combining (19) and (18). Equation (31) is obtained combining (5), (13), and (25), and using symmetry, (1), and (24) to simplify the resulting expression.
} 


$$
a_{t+1}=\mathcal{F}\left(k_{t}, n_{t}\right)+k_{t}(1-\delta) .
$$

Finally, the two side conditions to determine the equilibrium path are the transversality condition (21) and the initial stock of goods $a_{0}$.

\subsection{Existence of an Equilibrium}

The existence of an equilibrium is easily proved using the method that Stokey, Lucas, and Prescott (1989) denotes as the "indirect approach". The strategy of this indirect approach is to prove the existence of the optimal path of a pseudo-economy that matches the equilibrium path we are interested in. The following proposition formalizes this idea. A sketch of the proof is in the Appendix.

Proposition 1 An equilibirum where money is only held as a medium of exchange exists if $0<$ $R_{t}<\sigma^{2} /[2(1+\sigma)]$ for all $t \geq 0$ and $a_{0} \in(0, \widehat{a})$, where $\widehat{a}$ is implicitly defined by $\delta \widehat{a}=\mathcal{F}(\widehat{a}, 1)$. Moreover, the equilibrium path for $\left\{v_{t}, b_{t}, n_{t}, l_{t}, s_{t}, k_{t}, a_{t+1}\right\}_{t=0}^{\infty}$ in our model is identical to the optimal path of a pseudo-economy with the following characteristics: There is a representative consumer whose preferences are:

$$
\sum_{t=0}^{\infty} \beta^{t}\left[\omega_{1} \ln \left(v_{t}\right)+\omega_{2 t} \ln \left(b_{t}\right)+\mathcal{V}\left(l_{t}\right)\right]
$$

where

$$
\begin{gathered}
\omega_{1}=\frac{2}{2+\sigma} \text { and } \\
\omega_{2 t}=\frac{2}{2+\sigma} \frac{\sigma}{1-\sigma} \frac{1+\varphi_{t}}{\varphi_{t}} .
\end{gathered}
$$

The feasible paths are constrained by (6) to (8), (11), $s_{t}=\varphi_{t} b_{t}$, and the given initial stock of goods $a_{0}$.

The condition $0<R_{t}$ is necessary to ensure that constraint (12) is binding and so money is only held to be provided to buyers. The condition $R_{t}<\sigma^{2} /[2(1+\sigma)]$ is necessary to ensure that $b_{t}$ and $s_{t}$ are positive.

Proposition 1 not only proves the existence of an equilibrium but also provides a simple method to calculate it. To find an equilibrium path, we just need to solve a simple optimization program. ${ }^{11}$ This procedure should be especially useful for stochastic extensions of the model.

\footnotetext{
${ }^{11}$ To find the utility of the representative household, we must use (2) and (5) once the equilibrium path is found because utility levels differ between the two economies.
} 


\subsection{Dynamics}

For tractability, the analysis of the equilibrium dynamics are limited to the case in which $R_{t}$ is constant. In each period, the system of equations (8), (11), (24), (27), (29), (30), (31), and (32) determines the endogenous variables $x_{t}, q_{t}, b_{t}, s_{t}, l_{t}, n_{t}, k_{t}$, and $v_{t}$ as a function of $\mu_{t}$ and $a_{t}$. Using the implicit function theorem, the following proposition follows. (See the analysis of this dynamic system in the Appendix).

Proposition 2 The signs of the derivatives of the most important endogenous variables of the model with respect to $a_{t}$ and $\mu_{t}$ are summarized in the following panel:

\begin{tabular}{|c|c|c|c|c|c|c|c|c|c|c|c|c|}
\hline \multirow{3}{*}{\multicolumn{2}{|c|}{ Derivative with respect to: }} & \multicolumn{11}{|c|}{ Endogenous Variables } \\
\hline & & $x_{t}$ & $q_{t}$ & $b_{t}$ & $s_{t}$ & $l_{t}$ & $n_{t}$ & $k_{t}$ & $v_{t}$ & $y_{t+1}$ & $b_{t} / s_{t}$ & $k_{t} / n_{t}$ \\
\hline & & 0 & $?$ & + & + & - & + & + & - & + & 0 & $?$ \\
\hline & $a_{t}$ & 0 & + & - & - & - & + & 1 & 0 & + & 0 & + \\
\hline
\end{tabular}

For a given initial stock of goods $a_{t}$, an increase in the value of goods produced $\mu_{t}$ induces a shift of labor away from home services and into both market production and trading. Also, an increase in $\mu_{t}$ leads to a decline of goods for sale in favor of capital. For a given value $\mu_{t}$, an increase in $a_{t}$ is fully employed as capital. This atracts labor into the production of market goods from all other activities because of the complementarity between $k_{t}$ and $n_{t}$. Moreover, to continue exchanging the same amount of goods with fewer traders, the size of each transaction must increase.

The dynamic system has two stationary equations, one for $\mu_{t+1}=\mu_{t}$ and the other for $a_{t+1}=a_{t}$ :

$$
\begin{gathered}
\mathcal{F}_{k}\left(k_{t}, n_{t}\right)-\delta=\beta^{-1}-1 \\
\mathcal{F}\left(k_{t}, n_{t}\right)=v_{t}+\delta k_{t}
\end{gathered}
$$

Using the implicit function theorem, we can characterize the slope and the properties of the two lines described by these two equations (see the Appendix). In the plane $\left(\mu_{t}, a_{t}\right)$, the line $\left(a_{t+1}=a_{t}\right)$ is downward sloping. The line $\left(\mu_{t+1}=\mu_{t}\right)$ may be upward or downward sloping, but in any case its slope surpasses that of the line $\left(a_{t+1}=a_{t}\right)$. It can be shown using standard arguments that the two lines cross once and only once. The phase diagram of the system is represented in Figure 1. As the figure shows, the system is saddle path stable, and the stable arm is downward sloping. ${ }^{12}$

\footnotetext{
${ }^{12}$ Because the present model is in discrete time, convergence may not be monotonic. For monotonic convergence the smallest eigenvalue of the dynamic system must be between 0 and 1 . In general, checking this condition is analytically
} 
In the steady state, where the two stationary lines cross, the marginal product of capital is equal to the subjective discount rate.

During the process of accumulation of the stock of goods $a_{t}$, capital increases, and the value of an extra good produced $\mu_{t}$ falls. During this adjustment, the portion of goods destined for sale and consumption increases, the fractions of individuals engaged in trade, $b_{t}$ and $s_{t}$, fall, and the size of each transaction, $q_{t}$, increases. The effect of capital accumulation on the fraction of individuals destined to be production workers and home workers is ambiguous because the changes in $a_{t}$ and $\mu_{t}$ tend to move these variables in opposite directions.

\subsection{Balanced Growth Paths}

As in the neoclassical growth model, if technological change is labor augmenting, the economy converges to a balanced growth path. Let the production function have the form:

$$
y_{t}=\mathcal{F}\left(k_{t-1}, e_{t-1} n_{t-1}\right)
$$

where $e_{t}$ is the efficiency of labor in period $t$. Moreover, assume that the efficiency of labor grows at a constant rate $g: e_{t+1}=e_{t}(1+g)$. Then, a direct check of equations (24) to (34) that describe the dynamics of the model reveal that they are satisfied for a balanced path with the following properties: The variables $k_{t}, y_{t}, q_{t}$, and $\mu_{t}^{-1}$ are all growing at the rate $g$. In contrast, the variables $x_{t}, b_{t}, s_{t}, n_{t}$, and $l_{t}$ are constant. The stationary conditions are now:

$$
\begin{gathered}
\mathcal{F}_{k}\left(k_{t}, e_{t} n_{t}\right)-\delta=(1+g) \beta^{-1}-1 \text { and } \\
\mathcal{F}\left(k_{t}, e_{t} n_{t}\right)=(1+g) v_{t}+(g+\delta) k_{t}
\end{gathered}
$$

\subsection{The Demand for Money}

In an equilibrium where money is only wanted as a medium of exchange, (12) holds with equality. As in a cash-in-advance economy, the maximum quantity buyers can purchase in period $t$ is limited by the money held from period $t-1$ to period $t$. However, in the present model only a fraction of this money is spent in equilibrium. To spend their money, buyers must find a suitable seller that makes an acceptable offer. For this reason, the velocity of money with respect to the quantity of intractable. In the special case that $\mathcal{V}$ is linear, this condition can be easily checked around a steady state, and it is satisfied. (See the Appendix). In all numerical examples computed by the author with a logarithmic $\mathcal{V}$, convergence is also monotonic. 
goods actually purchased by households is not constant. Using (1), (9), (12), and the definition of $p_{t}$, we obtain:

$$
\text { Velocity at } t \equiv \frac{p_{t} v_{t}}{m_{t}}=x_{t} \frac{S_{t}}{B_{t}+S_{t}}
$$

The velocity of money with respect to $v_{t}$ (consumption expenditures) depends on how willing buyers are on departing from their most preferred variety and how easy it is for them to find a seller. Using (24) and (28), the following proposition follows.

Proposition 3 The velocity of money with respect to consumption expenditures in period $t$ is an increasing function of the opportunity cost of holding money from period $t-1$ to period $t$ :

$$
\text { Velocity at } t=\frac{\frac{\sigma^{2}}{1+\sigma}+R_{t}}{1.5 \sigma}
$$

\section{The Media of Exchange}

This section endogenizes the medium of exchange. In Section 2, fiat money is essential to trading for the combination of two factors. Firstly, barter arrangements are precluded by the preference structure. Secondly, commodity money is precluded by assuming that goods are not storable for over one period if they are not invested as capital. Both assumptions can be relaxed.

Barter can be easily introduced by changing the preference structure so two sellers could carry reciprocally desirable goods. This extension is straightforward but cumbersome, so it is not be pursued here.

Commodity money may exist if at least one good is durable even when it is not employed as capital in the production of other goods. With this assumption, there are many topics that can be studied. As in Kiyotaki and Wright (1989) one can assume that all goods are durable and each one of the $H$ goods depreciates at a different rate (or trades at a different utility cost). One can then study the conditions for a particular good to arise as a medium of exchange. In particular, one can inquire if a good with a relative high trading cost can be used as a medium of exchange. In the remaining of this section, the reasons why a particular physical commodity becomes the medium of exchange over another are simplified to concentrate on the choice between commodity money and fiat money.

One reasonable way of modelling commodity money is to assume that most goods have a high depreciation rate when they are employed as media of exchange, but there is one, to be referred to as gold, that neither depreciates nor is costly to carry and exchange. With this assumption, we 
have multiple equilibria of a sort familiar in search theoretic models. If all sellers demand payment in gold, it is optimal for a particular household to instruct its sellers to demand payment in gold to furnish its buyers with gold. But if all sellers demand dollars, it is optimal for a household to demand dollars. Consequently, the existence of an equilibrium with either medium of exchange is robust to a broad array of returns for gold and dollars. The medium of exchange can be said to depend on social custom.

Another way of introducing commodity money is to assume that all goods can be used as capital for all households, leaving the preference structure for the consumption of goods the same as in Section 2. With this assumption the acceptability of commodity money is assured, since all households have a good use for a particular good. Undoubtedly, this assumption exaggerates the liquidity of goods, but in doing so it provides a worst case scenario to investigate the conditions for the existence of equilibria where fiat money is the medium of exchange. To model an equilibrium with this assumption, I assume that sellers make offers conditional on the medium exchange brought by a buyer. Hence, they present to a buyer carrying dollars with an offer $\left(q_{t}, z_{t}\right)$, and they present to a buyer carrying goods with an offer $\left(\bar{q}_{t}, \bar{z}_{t}\right)$. As one would expect, when all goods are liquid, fiat money can only be a viable medium of exchange if its rate of return is not dominated by the rate of return on goods when they are used as media of exchange. More precisely, we obtain the following proposition.

Proposition 4 Suppose all goods can be used as capital for all households and depreciate at a common rate $\delta_{m}$ when used as media of exchange. Then, the medium of exchange used is the one with the lowest opportunity cost, which for fiat money is $R_{t} \equiv\left[1-\delta+\mathcal{F}_{k}\left(k_{t-1}, n_{t-1}\right)\right]\left(1+\pi_{t}\right)-1$, and for commodity money is $\rho_{t} \equiv\left[1-\delta+\mathcal{F}_{k}\left(k_{t}, n_{t}\right)\right]\left(1-\delta_{m}\right)^{-1}-1$. Consequently, in the steady state, fiat money is a viable medium of exchange if the gross rate of inflation $\left(1+\pi_{t}\right)$ does not exceed the gross rate at which commodity money depreciates $\left(1-\delta_{m}\right)^{-1}$.

The proof of Proposition 4 is in the Appendix.

\section{The Optimum Quantity of Money and Welfare}

This section analyzes substantive issues of monetary theory in an economy with fiat money: the interaction between the rate of growth of money and capital accumulation, the optimum quantity of money, the welfare properties of a monetary equilibrium, and the welfare costs of inflation. 


\subsection{Money and Capital Accumulation}

Money in the model is neutral. A once-and-for-all increase in the quantity of money distributed equally to all households increases monetary payments made in transactions proportionately without any real effect. Money, however, is not superneutral. Changes in the rate of growth of the money supply induce equal changes in the rate of inflation and thereby on the opportunity cost of holding money $R$. This subsection investigates how changes in $R$ affect capital accumulation and the other real variables in the economy. For tractability, this analysis is centered in a comparison across steady states. For brevity in notation, time subscripts are dropped.

In the system of equations that describes an equilibrium, the opportunity cost of holding money only enters equation (27). As implied by this equation, the direct effect of an increase in $R$ is a drop of the ratio $b / s$. This drop has an indirect effect on the other variables of the model as summarized in the following proposition. (For the proof see the comparative statics across steady states in the Appendix):

Proposition 5 The signs of the derivatives with respect to $R$ in comparisons across steady states are:

\section{Endogenous Variables}

\begin{tabular}{llllllllllllll}
\hline$x$ & $q$ & $b$ & $s$ & $l$ & $n$ & $k$ & $y$ & $v$ & $a$ & $\mu$ & $k / n$ & $s / n$
\end{tabular}

Derivative with respect to $R \quad 0+{ }_{-}+++++++{ }_{+}+0$ l 0

An increase in $R$ induces households to reduce the fraction of buyers they send to the market and to increase the quantity of goods purchased at each trade. The labor liberated with the drop in the number of buyers is spread over the three alternative activities in the model: $s, l$, and $n$. Since the ratio $k / n$ remains equal to the subjective discount rate, capital, output, and sales increase in the same proportion as $n$. In conclusion, an increase in $R$ due to an increase in the rate of growth of the money supply leads to an increase in the capital stock.

\subsection{The Optimum Quantity of Money}

This subsection investigates the conditions for the optimality of Friedman's (1969) prescription to reduce the opportunity cost of money to zero. Using (1), (2), and (5), together with symmetry, the effect of a small increase in $R$ on the one period utility of a representative household, to be denoted $W$, is:

$$
d W=\frac{1}{q} d q+\frac{1}{1-\sigma} \frac{b+s}{b s} d\left(\frac{b s}{b+s}\right)+\mathcal{V}^{\prime} d l
$$


Using the implicit function theorem results used to elaborate the previous table, we obtain the following derivative as long as $R$ is positive (see the comparative analysis across steady states in the Appendix):

$$
\frac{d W}{d R}=\frac{\left.\mathcal{V}^{\prime \prime}[(\sigma \varphi-1)(b+s)+\sigma \varphi n)\right]+\mathcal{V}^{\prime}\left[\mathcal{V}^{\prime}(b+s)(1-\sigma)-\sigma \varphi\right]}{(1-\sigma) \varphi(1+\varphi)\left[\mathcal{V}^{\prime}-\mathcal{V}^{\prime \prime}(1-l)\right]}
$$

The denominator in (46) is always positive. Hence, the condition for $R \rightarrow 0^{13}$ to maximize steady state utility is a negative numerator. The second summand of the numerator is always negative (see the Appendix). Hence, a sufficient condition for the numerator to be negative is:

$$
\mathcal{V}^{\prime \prime}[(\sigma \varphi-1)(b+s)+\sigma \varphi n] \leq 0
$$

Condition (47) is satisfied in case $\sigma \geq 0.5$ because $\varphi \geq 2$ (see equation [28]) and $\mathcal{V}^{\prime \prime} \leq 0$. Consequently, $d W / d R$ is negative if the desire for diversity is strong $(\sigma \geq 0.5)$, the supply of labor is perfectly elastic $\left(\mathcal{V}^{\prime \prime}=0\right)$, or the total number of traders $(b+s)$ is not too large relative to producers $(n)$.

Conversely, the derivative $d W / d R$ to be positive requires a low $\sigma$ and hence a low markup $(1-\sigma)^{-1}$. Moreover, it requires a large fraction of traders relative to producers. When $\sigma$ is low, the fraction of traders relative to producers can only be large if traders take a long time to meet one another, that is if the length of the period is long. For example, with the baseline parameters of the following subsection (except for $\sigma$ and $T$ ), the length of the period must be over 78 years $(T<1 / 78)$ for $d W / d R>0$ if the markup is a minute 1.01. The length of the period must be over 544 years for the same purpose if the markup is 1.1. For realistic markups 1.2 and over, there is no period shorter than one million years that yields $d W / d R>0$.

As long as $R$ remains positive and $d W / d R$ is negative, reducing $R$ improves steady state utility. Transitional dynamics further reinforce the benefits of reducing $R$ because this reduction leads to an economy with a lower capital stock. Therefore, with a lower $R$ households enjoy not only a higher steady state utility, but also the benefit of having to invest little during the transition to the new steady state. In conclusion, we obtain the following proposition:

Proposition 6 The optimum opportunity cost of holding money is zero if

$$
\left.\mathcal{V}^{\prime \prime}[(\sigma \varphi-1)(b+s)+\sigma \varphi n)\right]+\mathcal{V}^{\prime}\left[\mathcal{V}^{\prime}(b+s)(1-\sigma)-\sigma \varphi\right]<0,
$$

\footnotetext{
${ }^{13}$ When $R=0$, the constraint (12) is not binding which opens the possibility to multiple equilibria. With $R \rightarrow 0$, I indicate that of these equilibria I use the one where money is not held as store of value for the comparisons across steady states in this section.
} 
which is satisfied for reasonable parameters. ${ }^{14}$

\subsection{Optimal Allocations}

This subsection studies the welfare properties of the equilibrium paths described in Section 2. To this end, it characterizes the symmetric optimal path that maximizes the utility of a representative household when the decisions about the extend of search $x_{t}$, production $q_{t}$, investment $\left(k_{t}-k_{t-1}\right)$, and labor allocation $\left(b_{t}, s_{t}, n_{t}, l_{t}\right)$ are made by a benevolent central authority. Following standard practice in this literature, this central authority is not bound to using money in exchange. However, the central authority must abide to the resources available in the economy, the bilateral matching among traders, and a single quantity delivered to all purchasing buyers.

The symmetric optimal path maximizes the following objective:

$$
\sum_{t=0}^{\infty} \beta^{t}\left\{q_{t}\left[\mathcal{M}\left(b_{t}, s_{t}\right)\left(x_{t}-\frac{x_{t}^{2}}{2}\right)\right]^{\frac{1}{1-\sigma}}+\mathcal{V}\left(l_{t}\right)\right\},
$$

where $\mathcal{M}\left(b_{t}, s_{t}\right)=\mathcal{B}\left(b_{t}, b_{t}, s_{t}\right)=\mathcal{S}\left(s_{t}, b_{t}, s_{t}\right)$ is the probability that a buyer meets a seller in the appropriate market. The following two constraints bind the optimal path:

$$
\begin{gathered}
\mathcal{F}\left(k_{t-1}, n_{t-1}\right)+(1-\delta) k_{t-1}-q_{t} x_{t} \mathcal{M}\left(b_{t}, s_{t}\right)-k_{t}=0 \text { and } \\
1-l_{t}-n_{t}-b_{t}-s_{t}=0
\end{gathered}
$$

together with the non-negativity constraints on the choice variables: $x_{t}, q_{t}, b_{t}, s_{t}, n_{t}, l_{t}$, and $k_{t}$.

Denoting $\mu_{t} \beta^{t}$ the Lagrange multiplier for constraint (49), the first order conditions for an interior solution of the optimal paths are:

$$
\begin{gathered}
\mathcal{U}^{\prime}\left(c_{t}\right) c_{t}^{\sigma} \frac{q_{t}^{1-\sigma}}{1-\sigma}\left(1-x_{t}\right)=\mu_{t} q_{t}, \\
\mathcal{U}^{\prime}\left(c_{t}\right) c_{t}^{\sigma} q_{t}^{-\sigma}\left(1-\frac{x_{t}}{2}\right)=\mu_{t}, \\
\mathcal{U}^{\prime}\left(c_{t}\right) c_{t}^{\sigma} \frac{q_{t}^{1-\sigma}}{1-\sigma}\left(x_{t}-\frac{x_{t}^{2}}{2}\right) \mathcal{M}_{b}\left(b_{t}, s_{t}\right)=\mu_{t} q_{t} x_{t} \mathcal{M}_{b}\left(b_{t}, s_{t}\right)+\mathcal{V}^{\prime}\left(l_{t}\right), \\
\mathcal{U}^{\prime}\left(c_{t}\right) c_{t}^{\sigma} \frac{q_{t}^{1-\sigma}}{1-\sigma}\left(x_{t}-\frac{x_{t}^{2}}{2}\right) \mathcal{M}_{s}\left(b_{t}, s_{t}\right)=\mu_{t} q_{t} x_{t} \mathcal{M}_{s}\left(b_{t}, s_{t}\right)+\mathcal{V}^{\prime}\left(l_{t}\right), \\
\mu_{t+1} \beta \mathcal{F}_{n}\left(k_{t}, n_{t}\right)=\mathcal{V}^{\prime}\left(l_{t}\right), \text { and }
\end{gathered}
$$

\footnotetext{
${ }^{14}$ To achieve $R \rightarrow 0$, both inflation and the rate of growth of the money supply must be negative. The lump-sum taxes necessary to implement this policy could be problematic and counterproductive if households were heterogeneous.
} 


$$
\mu_{t+1} \beta\left[1-\delta+\mathcal{F}_{k}\left(k_{t}, n_{t}\right)\right]=\mu_{t}
$$

Conditions (55) and (56) are identical to the analogous conditions for a representative household in the equilibrium of Section 2. Hence, the search environment does not affect directly the margin of choices for capital and labor in market production. This production efficiency property is the trademark of the neoclassical growth model. The remaining conditions for optimality have profound differences from their counterparts in an equilibrium. The utility of consuming one good of the reservation variety, $x_{t}$, is now equated to the marginal value of producing one good instead of the marginal value of purchasing one (condition [51]). Remember that these two marginal values differ in equilibrium because of the ex-post monopoly power of sellers. The marginal utility of increasing $q_{t}$ is now equated to the value of a good produced without regard to the effect this has on the number of sales (condition [52]). Finally, the utility brought by the goods transacted by the marginal trader is now equated to the cost of producing the extra goods transacted plus the opportunity cost of the traders time (conditions [53] and [54]).

Equations (51) and (52) imply:

$$
x_{t}=\frac{2 \sigma}{1+\sigma}
$$

In an optimal allocation, the range of acceptable varieties is double the equilibrium range. In equilibrium, buyers do not internalize the benefit they bring to sellers when they accept an offer, so buyers are too picky when they decide on accepting a variety. Combining (53) and (54), we obtain:

$$
\mathcal{M}_{b}\left(b_{t}, s_{t}\right)=\mathcal{M}_{s}\left(b_{t}, s_{t}\right)
$$

This implies $b_{t}=s_{t}$, which maximizes the meeting between buyers and sellers. In contrast in equilibrium, $b_{t} \leq(1 / 2) s_{t}$.

In the steady state, the optimal allocation can be closely compared to an equilibrium allocation with $R \rightarrow 0$. Key variables and ratios are compared in the following equations where an asterisk denotes optimal allocation and the absence of an asterisk denotes equilibrium (see the Appendix for their derivation):

$$
\begin{gathered}
\frac{k}{n}=\frac{k^{*}}{n^{*}}, \\
x=\frac{1}{2} x^{*}, \\
\frac{s}{n}=2 \frac{s^{*}}{n^{*}}, \\
\frac{b}{s}=\frac{1}{2} \frac{b^{*}}{s^{*}}=\frac{1}{2}, \text { and }
\end{gathered}
$$




$$
q=\frac{3}{2} q^{*}
$$

In equilibrium, the capital-labor ratio is efficient. However, the following margins of choice are distorted from an optimal allocation: Households are too picky about the goods they buy. Too many workers are selling market goods relative to both the workers that are producing these goods and the buyers that are purchasing them. Finally, the size of transactions is too large. Increasing $R$ has no effect on the allocation margins compared in (59) to (61), but it exacerbates the two distortions in (62) and (63). That is, an increase in $R$ further depresses $b / s$ and increases $q$. In contrast, increasing $R$ may improve the allocation between traders and either producers or home workers or both. For example, when $\mathcal{V}(l)=\theta \ln l$, we obtain

$$
\frac{b+s}{l}=\frac{3}{2+\sigma} \frac{b^{*}+s^{*}}{l^{*}}
$$

Since $\sigma \in(0,1)$, there are too many traders in equilibrium relative to home workers. Increasing $R$ reduces $b+s$ and increases $l$, so it improves this allocation margin. This explains why there may be instances in which steady state welfare is maximized with a positive $R$. However, the direct effect of an increase in $R$ is to reduce $b / s$, which is counterproductive, so it is not surprising that under mild conditions $R \rightarrow 0$ is optimal.

\subsection{The Merchant's Dilemma}

Could an optimal allocation be decentralized as a monetary equilibrium with a different bargaining solution other than giving full power to sellers to make take-it-or-leave-it offers? The answer is no as long as there is commerce and buyers pay only for the products they acquire.

As long as buyers choose $x_{t}$, the first order condition (13) applies. Comparison of this condition with (51) implies that for efficiency there should be no markup between the value of producing $q_{t}$ and the value received as payment when selling $q_{t}$, that is $\mu_{t} q_{t}$ must be equal to $\lambda_{t} z_{t}$. In principle, if buyers had the power to make take-it-or-leave-it offers to sellers, this would be the outcome. Nevertheless, the following old merchant's dilemma would also arise: "If you sell at cost, you work for free." In fact, when $\mu_{t} q_{t}=\lambda_{t} z_{t}$, the equilibrium condition (16), which must apply in an interior choice of $s_{t}$, can never hold. The opportunity cost of one seller's labor always exceeds the gains the seller brings to the household. Hence, $s_{t}$ must be at the corner solution zero. For sellers to

exist, they must be able to cover not only the cost of producing what they sell, but also the cost of servicing sales (successful or not) and the cost of waiting for customers. 
The merchant dilemma is not circumscribed to random matching. It applies as long as the matching functions $\mathcal{B}$ and $\mathcal{S}$ require sellers in the commerce sector. Conversely, if $\mathcal{B}\left(b_{t}, B_{t}, S_{t}\right)=b_{t}$ and $\mathcal{S}\left(b_{t}, B_{t}, S_{t}\right)=B_{t}$, the optimal allocation can be decentralized with take-or-leave-it offers by buyers and $R_{t} \rightarrow 0$. However, this eliminates the commercial sector, whereas in the United States the value added generated in commerce is over 80 percent of the joint value added of agriculture, forestry, fishing and manufacturing. ${ }^{15}$ The main point of this section is summarized in the following proposition:

Proposition 7 An optimal allocation can be decentralized as a monetary equilibrium if and only if $R_{t} \rightarrow 0$, buyers make take-or-leave-it offers to sellers, and successful matching rates are independent from the fraction of sellers, so there is no commerce in equilibrium.

\subsection{The Welfare Cost of Inflation}

This subsection calculates the welfare cost of inflation in a numerical evaluation of the model. The baseline evaluation is reported in Table 1. For this evaluation, most functional forms and parameters are standard. ${ }^{16}$ The production function is Cobb-Douglas with 0.36 capital share. The annual depreciation rate is 0.10 . Labor efficiency grows at 0.018 per year. The discount factor is set to produce a 0.04 annual real rate of interest in the steady state. Both the utility of consumption and home services are logarithmic. The weight in home services is set to induce households to spend 46 percent of their working time at home either purchasing goods or producing home services in a steady state with zero inflation. ${ }^{17}$ The preference for diversity is set to generate a 1.4 ratio between the price paid by households and the marginal cost of production (commercial markup). This estimate is proposed by Rotemberg and Woodford (1995) and it has been adopted in other monetary models with goods' diversity (see, for example, Christiano, Eichenbaum, and Evans [2001]) . Finally, the length of the period is set so the annual velocity of money in a steady

\footnotetext{
${ }^{15}$ The BEA estimates in billions of dollars for 1999 are agriculture, forestry and fishing 109.2, manufacturing 1193.3, wholesale trade 441.8, and retail trade 635.2. These figures were obtained March 23, 2001 at www.bea.doc.gov.

${ }^{16}$ See, for example, Cooley and Hansen (1989) numerical evaluation of the inflation tax. Apart from the absence of bilateral matching and the preference for consumption diversity, Cooley and Hansen (1989) differ from the present work by abstracting from growth, using a linear utility of leisure, and only comparing welfare across steady states.

${ }^{17}$ Accoding to Benhabib, Rogerson, and Wright (1991) households spend 0.33 of their time working in the market (selling or producing market goods) and 0.28 working at home (buying or producing home services). The ratio $0.28 /(0.28+0.33)$ is equal to 0.46 . These estimates are elaborated from the analysis in Hill (1985) of the Michigan Time Use Survey.
} 
state at zero inflation is 11.34 . This is the annual velocity of M0 with respect to consumption expenditures in the United States. ${ }^{18}$

The annual velocity of money in the model depends mainly on the length of the period. The shorter the period the higher the velocity is. To match the observed M0 velocity in the United States, the period must be around 4.8 days (76 periods per year). This short period is a good feature of the model. In comparison, models with a cash-in-advance constraint have a unit velocity per period so they require periods of 32 days (11.34 periods per year) to match the same annual velocity. Such a long period is problematic. One thing is to require individuals to use cash to buy goods, but quite a different one is to make them earn their cash 32 days in advance of the time they can spend it.

Table 1 shows the allocation of labor among the four different activities in the model with the baseline parameters. The fraction of sellers to producers is around 44 percent. This fraction is large by comparison with most macroeconomic models where it is zero. However, in the United States employment in commerce (retail and wholesale trade) represents 38 percent of the rest of private employment. And it represents 114 percent of the employment in goods' producing industries (agriculture, mining, manufacturing, and construction). ${ }^{19}$

As shown in Table 1, an increase in inflation reallocates buyers to the three other activities in the model. This reallocation increases the velocity of money and worsens welfare. The welfare cost of inflation is measured as the compensating variation on the initial stock of goods $a_{t}$ when inflation drops from the rate we are considering $(\pi)$ to zero. That is, we find the maximum reduction in $a_{t}$ that households are willing to suffer to get inflation permanently dropped from $\pi$ to zero. This calculation takes into account the whole transition path to the new steady state, which was calculated using standard numerical methods. ${ }^{20}$ In Table 1 , the welfare cost of inflation is expressed as a percentage of the present value of lifetime market consumption expenditures (global wealth) in the original equilibrium. As one can observe, the welfare cost of inflation is substantial. The cost of a 10 percent rate of inflation is almost 0.7 percent of global wealth. Also, the cost of having

\footnotetext{
${ }^{18}$ This figure refers to seasonally adjusted data in the last quarter of 2000 . The original data was obtained from www.federalreserve.gov/releases and www.bea.doc.gov. The velocity of M1 with respect to consumption expenditures in the same period was 6.07 .

${ }^{19}$ In December 2000, total private nonfarm employment was 111.443 million of those 25.569 million worked in goods producing industries and 31.330 million worked in retail and wholesale trade. In the same month, employment in agriculture was 3.274 million. All these figures are seasonally adjusted. Source: stats.bls.gov/new.release/empsit.t01.htm and stats.bls.gov/new.release/empsit.t11.htm, downloaded May 25, 2001.

${ }^{20}$ The calculations were done using Gauss. The programs are available from the author upon request.
} 
zero inflation instead of implementing the Friedman rule is an extra 0.25 percent of global wealth.

To bring these costs into a practical perspective, I report in the last row of Table 1 the net benefit of having a national currency as the medium of exchange. The calculation of this net benefit assumes that the national currency entails an inflation $\pi$ and there is an alternative form of money that would bring price stability. This type of cost-benefit analysis was advocated by Friedman (1986) in an article where he questions the superiority of irredeemable fiat money over gold as the medium of exchange. When gold is used as the medium of exchange, a country gives up the return of this gold in industrial uses. The present value of these returns for an infinite horizon is the value of the currency in circulation. Therefore, the net benefit of a national currency is the value of the currency in circulation with price stability minus the welfare cost of the inflation the national currency brings. A similar calculation applies to countries that adopt the US dollar as their medium of exchange to control inflation ("dollarization") as several countries have recently done. In this instance, the country that adopts the US dollar gives up the return of investing the circulating US dollars in assets such as US Treasury Bills. As shown in the last row of Table 1, if inflation is less than 2.9 percent, the net benefit of the national currency is positive. However, for inflations above 2.9 percent, it is better to adopt gold or a foreign currency that brings price stability rather than to keep the national currency. (This cost-benefit analysis does not include the value of the pride of having a national currency and the costs of uncertain inflation).

Table 2 reproduces the type of estimates of Table 1 in a standard model with a cash-in-advance constraint. $^{21}$ Often this constraint is informally motivated with bilateral matching. However, the estimates of the welfare cost of inflation in Table 2 are trivial compared to those in Table 1 . With a cash-in-advance constraint, the welfare cost of a 10 percent rate of inflation is a mere 0.003 percent of global wealth. Consequently, the net benefit of maintaining a fiat national currency is must higher with a cash-in-advance constraint than with bilateral matching. With cash-in-advance, as long as inflation is below 112 percent, it is preferable keeping the national currency rather than "dollarizing" or adopting gold. ${ }^{22}$

\footnotetext{
${ }^{21}$ The models of Tables 1 and 2 share the same production function and the same discount factor. In the model of Table 2, there is a single good and labor has two uses. The coefficient of home work in the utility function and the length of a period have been adjusted to produce the same annual velocity and fraction of individuals at home in the two tables when inflation is zero.

${ }^{22}$ The welfare costs of inflation reported in Table 2 are much smaller than those in Cooley and Hansen (1989). The main reason for the disparity is the absence of transitional costs of accumulating capital in Cooley and Hansen. (With cash-in-advance low inflation means high steady state capital). With the parameters of Cooley and Hansen's monthly model, the welfare cost of a 10 percent rate of inflation is 0.152 percent when transitional costs are omitted.
} 
Table 3 performs a sensitivity analysis of the cost of inflation to alternative parameter values in the bilateral matching model. The table calculates the same information of Table 1 for alternative real interest rates, commercial markups, and targeted velocities of money.

The real interest rate is dropped to 2 percent in column (1) and increased to 6.5 percent in column (2). Rates comprised by the broad interval from 2 to 6.5 percent are commonly used in quantitative macroeconomic models. This diverse practice is due to the wide disparity of observed real returns on assets that differ on risk and liquidity, features that are abstracted in the present model. Fortunately, we observe in Table 3 that the value of the real interest rate has little effect on the welfare cost of inflation. In both columns (1) and (2), the welfare cost of inflation remains around 0.7 percent of global wealth.

The commercial markup is increased in column (3) to 1.8 and dropped in column (4) to 1.2. On the one hand, some papers with diversified products have defended a 1.2 ratio between price and marginal cost (see, for example, Hornstein [1993] and Alexopoulos [2000]). On the other hand, many commercial products such as clothing operate with commercial margins well above the baseline 40 percent of Table 1. We observe in Table 3 that the larger is the commercial markup the larger is the ratio between sellers and producers of market goods. For example, with a 1.2 commercial markup the ratio $s / n$ is 22 percent, while with a 1.8 markup the ratio $s / n$ reaches 87 percent. Likewise, a drop in the commercial markup reduces the welfare cost of inflation. However, the welfare cost of a 10 percent rate of inflation remains above 0.6 percent of global wealth with a 1.2 commercial markup.

The velocity of money is targeted to be that of M1 in column (5) and double the observed velocity of M0 in column (6). In reality, deposits are a widely used medium of exchange and those included in M1 earn almost no interest. In contrast, some of M0 is not used as a regular medium of exchange in the United States. In fact, some economists calculate that half of the US currency is used by foreigners or in illegal activities. ${ }^{23}$ As expected, the higher is the velocity of money the lower are the costs of inflation. Roughly, when half the money is used in transactions, the welfare cost of inflation is also halved. For example, when we assume that the velocity of money is twice the apparent velocity of $\mathrm{M} 0$, the welfare cost of a 10 percent rate of inflation drops to a still substantial 0.325 percent of global wealth.

Whereas, the equivalent welfare cost is 0.006 percent when transitions taken into account.
${ }^{23}$ See Rogoff (1998). Also, see Camera (1999) for a search model with hidden activities. 


\section{Conclusion}

In the real world we observe that most exchange of final products is realized in large commercial sectors with bilateral trades. This important feature of reality is abstracted in models with Walrasian markets such as the neoclassical growth model. The present paper advances a model with bilateral matching in a neoclassical growth framework. This model not only motivates the existence of money but also accounts for the presence of a large commercial sector.

The model advanced in this paper has the following properties: Manufacturing efficiently combines capital and labor to obtain output with the delay of one period. During each period, the predetermined stock of goods is commercially exchanged by buyers and sellers that are paired bilaterally either randomly or according to a more general matching function. Prices are set by sellers. Money plays a useful role as media of exchange due to the rare double coincidence of wants. The objects that emerge as money can be determined endogenously. Both money and goods are perfectly divisible.

Despite the many features it has, the model remains tractable and suitable as a framework for investigating many topics in monetary economics. In this paper, the model is applied to the study of the effects of inflation on capital accumulation and welfare. It is found that inflation raises capital and output as a result of a shift of resources from commerce to manufacturing. Also, with realistic parameters inflation has large negative effects on welfare. In fact, for reasonable parameters "dollarization" passes a cost-benefit analysis for countries that cannot control even moderate rates of inflation with their national currencies. In contrast, with cash-in-advance, a device informally motivated with bilateral trading, inflation depresses capital and output and has a negligible effect on welfare.

Although it is not attempted in this paper, the model could provide a new perspective for nominal non-neutralities stemming from limited participation in financial markets and price rigidities. Bilateral trading provides a natural environment for costs in the adjustment of financial portfolios which are the driving force in models with limited participation in financial markets. Likewise, the existence of a commercial sector with a degree of indeterminacy in prices provides a natural environment to model price rigidities. 


\section{References}

[1] Alexopoulos, Michelle, "Shirking in a Monetary Business Cycle Model," 2000, manuscript.

[2] Alvarez, Fernando, and Nancy L. Stokey, "Dynamic Programming with Homogeneous Functions," Journal of Economic Theory 82, 1998, 167-189.

[3] Camera, Gabriele, "Dirty Money," 1999, manuscript.

[4] Cooley, Thomas F, and Gary D. Hansen, "The Inflation Tax in a Real Business Cycle Model," American Economic Review 89 (4), 1989, 733-748.

[5] Christiano, Lawrence J., Martin Eichenbaum, and Charles Evans, "Nominal Rigidities and the Dynamic Effects of a Shock to Monetary Policy," 2001 manuscript.

[6] Friedman, Milton, "The Optimum Quantity of Money," in The Optimum Quantity of Money, 1969, 1-69.

[7] Friedman, Milton, "The Resource Cost of Irredeemable Paper Money," Journal of Political Economy 94 (3) Part 1, 1986, 642-647.

[8] Hornstein, Andreas, "Monopolistic Competition, Increasing Returns to Scale and the Importance of Productivity Shocks," Journal of Monetary Economics 31, 1993, 299-316.

[9] Kiyotaki, Nobuhiro, and Randall Wright, "On Money as a Medium of Exchange," Journal of Political Economy 97, 1989, 927-954.

[10] Kiyotaki, Nobuhiro, and Randall Wright, "A Search-Theoretic Approach to Monetary Economics," American Economic Review 83 (1), March 1993, 63-77.

[11] Laing, Derek, Victor E. Li, and Ping Wang, "Inflation, Trade Frictions, and Productive Activity in a Multiple-Matching Model of Money," Working Paper 2000-28, Federal Reserve Bank of Atlanta, November 2000.

[12] Li, Victor, "The Optimal Taxation of Fiat Money in Search Equilibrium," International Economic Review 36(4), November 1995, 927-942.

[13] Molico, Miguel, "The Distribution of Money and Prices in Search Equilibrium," 1998, manuscript. 
[14] Rogoff, Ken, "Blessing or Curse? Foreign and Underground Demand for Euro Notes," Economic Policy, April 1998, 262-303.

[15] Rotemberg, Julio, and Michael Woodford, "Dynamic General Equilibrium Models with Imperfectly Competitive Product Markets." In Frontiers of Business Cycle Research, ed. Thomas F. Cooley. Princeton: Princeton University Press, 1995.

[16] Shi, Shouyong, "Money and Prices: A Model of Search and Bargaining," Journal of Economic Theory 67, 1995, 467-496.

[17] Shi, Shouyong, "A Divisible Search Model of Fiat Money," Econometrica 65(1), January 1997, 75-102.

[18] Shi, Shouyong, "Search, Inflation, and Capital Accumulation," Journal of Monetary Economics 44, 1999, 81-103.

[19] Shi, Shouyong, "Liquidity, Bargaining, and Multiple Equilibria in a Search Monetary Model," Annals of Economics and Finance 2, 2001, 191-197.

[20] Soller-Curtis, Elisabeth, and Randall Wright, "Price Setting and Price Dispersion in a Monetary Economy; or, the Law of Two Prices," manuscript Department of Economics University of Pennsylvania, January 2000.

[21] Stokey, Nancy L. and Robert E. Lucas Jr. with Edward C. Prescott, Recursive Methods in Economic Dynamics. Harvard University Press: Cambridge, Massachusetts, and London, England, 1989.

[22] Taber, Alexander and Neil Wallace, "A Matching Model with Bounded Holdings of Indivisible Money," International Economic Review 40(4), 1999, 961-984.

[23] Trejos, Alberto, and Randall Wright, "Search, Bargaining, Money, and Prices," Journal of Political Economy 103(1), 1995, 118-141.

[24] Zhou, Ruilin, "Individual and Aggregate Real Balances in a Random-Matching Model," International Economic Review 40(4), 1999, 1009-1038. 


\section{APPENDIX}

\section{Proof of Proposition 1 (Sketch)}

With the assumption $R_{t}<\sigma^{2} /[2(1+\sigma)]$, both $\varphi_{t}$ and $\alpha_{2 t}$ are positive. We can restrict $a_{t} \leq \widehat{a}$, where $\widehat{a}$ is implicitly defined by $\delta \widehat{a}=\mathcal{F}(\widehat{a}, 1)$, because $a_{t}$ can never reach $\widehat{a}$ if $a_{0}<\widehat{a}$. With this restriction the objective in (35) is bounded above in the set of feasible paths. Moreover, as long as $a_{0}>0$, there is at least one feasible path for which the objective in (35) is greater than $-\infty$. Consequently, there is a solution to the optimization program. ${ }^{24}$ This solution is unique because the objective is concave and the restrictions are convex. Moreover, this solution is characterized with the first order conditions of the program. These first order conditions are a reduced form of the set of equations that characterizes an equilibrium for the original economy. Therefore, an equilibrium for the latter economy exists.

With $\omega_{1}, \omega_{2 t}$, and $\varphi_{t}$ positive, $v_{t}, b_{t}$, and $s_{t}$ must be positive for all $t$. Likewise, the Inada conditions assumed on $\mathcal{F}$ and $\mathcal{V}$ imply that $k_{t}, n_{t}$, and $l_{t}$ are positive. Finally, (31) implies that $q_{t}$ must be positive as well. Consequently, the equilibrium is diversified and monetary.

\section{Analysis of the dynamic system of Section 2}

In abbreviated form, the system of equations that determines $s, l, n$, and $k$ can be written as

$$
\begin{aligned}
(a-k) \mu-\alpha_{1} & =0, \\
1-\alpha_{2} s-n-l & =0, \\
\mathcal{V}^{\prime}(l) s-\alpha_{3} & =0, \text { and } \\
\Phi\left(\frac{k}{n}\right) \mu-\mathcal{V}^{\prime}(l) & =0 .
\end{aligned}
$$

This system is an abbreviated version of (8) and (32), (11) and (28), (29), and (30), where $\Phi(k / n)=$ $\mathcal{F}_{n} /\left(1-\delta+\mathcal{F}_{k}\right)\left(\Phi^{\prime}>0\right)$, and $\alpha_{1}, \alpha_{2}$, and $\alpha_{3}$ are expressions independent of $k$ and $\mu$. These expressions are constant when $R$ is constant. Differentiating this system, we obtain ${ }^{25}$ :

$$
\left[\begin{array}{cccc}
0 & 0 & 0 & -\mu \\
-\alpha_{2} & -1 & -1 & 0 \\
\mathcal{V}^{\prime} & \mathcal{V}^{\prime \prime} s & 0 & 0 \\
0 & -\mathcal{V}^{\prime \prime} & -\Phi^{\prime} k \mu n^{-2} & \Phi^{\prime} n^{-1} \mu
\end{array}\right]\left[\begin{array}{c}
d s \\
d l \\
d n \\
d k
\end{array}\right]=\left[\begin{array}{c}
-v \\
0 \\
0 \\
-\mathcal{V}^{\prime} \mu^{-1}
\end{array}\right] d \mu+\left[\begin{array}{c}
-\mu \\
0 \\
0 \\
0
\end{array}\right] d a
$$

\footnotetext{
${ }^{24}$ See Alvarez and Stokey (1998, Sections 4 and 5) for a detailed argument.

${ }^{25}$ These derivatives have been simplified using (65) to (68) and $v=\alpha_{1} \mu^{-1}$.
} 
Solving this equation, ${ }^{26}$ we obtain

$$
\begin{gathered}
{\left[\begin{array}{l}
d s \\
d l \\
d n \\
d k
\end{array}\right]=A_{1}^{-1}\left[\begin{array}{c}
s n V^{\prime \prime}\left(v \mu \Phi^{\prime}+n \mathcal{V}^{\prime}\right) \\
-n V^{\prime}\left(v \mu \Phi^{\prime}+n \mathcal{V}^{\prime}\right) \\
n\left(v \mu \Phi^{\prime}+n \mathcal{V}^{\prime}\right)\left[\mathcal{V}^{\prime}-(b+s) \mathcal{V}^{\prime \prime}\right] \\
v \mu^{-1} A_{1}
\end{array}\right] d \mu, \text { and }} \\
{\left[\begin{array}{c}
d s \\
d l \\
d n \\
d k
\end{array}\right]=\mathcal{V}_{1}^{-1}\left[\begin{array}{c}
\prime \\
-\mathcal{V}^{\prime} \Phi^{\prime} n \mu^{2} \\
{\left[\mathcal{V}^{\prime}-(b+s) \mathcal{V}^{\prime \prime}\right] \Phi^{\prime} n \mu^{2}} \\
A_{1}
\end{array}\right] d a .}
\end{gathered}
$$

where $A_{1}=\left[\mathcal{V}^{\prime}-(b+s) \mathcal{V}^{\prime \prime}\right] \Phi^{\prime} k \mu^{2}-n^{2} \mathcal{V}^{\prime} \mathcal{V}^{\prime \prime} \mu>0$. Using the definitions of $v$ and $y$,

$$
\begin{gathered}
{\left[\begin{array}{c}
d q \\
d(k / n) \\
d v \\
d y
\end{array}\right]=A_{1}^{-1}\left[\begin{array}{c}
-q\left[s \mu^{-2} A_{1}-\mathcal{V}^{\prime \prime} n\left(\mathcal{V}^{\prime} n+\Phi^{\prime} v \mu\right)\right] \\
-\mathcal{V}^{\prime}\left\{v n \mathcal{V}^{\prime \prime}+k\left[\mathcal{V}^{\prime}-(b+s) \mathcal{V}^{\prime \prime}\right]\right\} \\
-v \mu^{-1} A_{1} \\
\mathcal{F}_{n} n\left(v \mu \Phi^{\prime}+n \mathcal{V}^{\prime}\right)\left[\mathcal{V}^{\prime}-(b+s) \mathcal{V}^{\prime \prime}\right]+\mathcal{F}_{k} v \mu^{-1} A_{1}
\end{array}\right] d \mu \text {, and }} \\
{\left[\begin{array}{c}
d q \\
d(k / n) \\
d v \\
d y
\end{array}\right]=A_{1}^{-1}\left[\begin{array}{c}
-q n \Phi^{\prime} \mathcal{V}^{\prime \prime} \mu^{2} \\
-\mathcal{V}^{\prime} \mathcal{V}^{\prime \prime} n \mu \\
0 \\
\mathcal{F}_{n} n \Phi^{\prime} \mu^{2}\left[\mathcal{V}^{\prime}-(b+s) \mathcal{V}^{\prime \prime}\right]+\mathcal{F}_{k} A_{1}
\end{array}\right] d a .}
\end{gathered}
$$

Using (38), the slope of the stationary line $\left(\mu_{t}=\mu_{t+1}\right)$ is:

$$
\left(\frac{d a}{d \mu}\right)_{\mu_{t}=\mu_{t+1}}=-\frac{\frac{d\left(\frac{k}{n}\right)}{\frac{d \mu}{d\left(\frac{k}{n}\right)}}}{d a}=-\frac{v}{\mu}+\frac{k}{n} \frac{\left[\mathcal{V}^{\prime}-(b+s) \mathcal{V}^{\prime \prime}\right]}{-\mathcal{V}^{\prime \prime} \mu} .
$$

Likewise, using (39), the slope of the stationary line $\left(a_{t}=a_{t+1}\right)$ is:

$$
\begin{aligned}
\left(\frac{d a}{d \mu}\right)_{a_{t}=a_{t+1}}= & -\frac{\frac{d v}{d \mu}-\delta \frac{d k}{d \mu}-\frac{d y}{d \mu}}{\frac{d v}{d a}-\delta \frac{d k}{d a}-\frac{d y}{d a}}= \\
& -\frac{v}{\mu} \frac{(k+v)\left[\mathcal{V}^{\prime}-(b+s) \mathcal{V}^{\prime \prime}\right] \Phi^{\prime} \mu-\left(1+\mathcal{F}_{k}-\delta\right) n^{2} \mathcal{V}^{\prime} \mathcal{V}^{\prime \prime}}{v\left[\mathcal{V}^{\prime}-(b+s) \mathcal{V}^{\prime \prime}\right] \Phi^{\prime} \mu-\left(\mathcal{F}_{k}-\delta\right) n^{2} \mathcal{V}^{\prime} \mathcal{V}^{\prime \prime}} \\
& -\frac{\mathcal{F}_{n} n^{2} \mathcal{V}^{\prime}\left[\mathcal{V}^{\prime}-(b+s) \mathcal{V}^{\prime \prime}\right]}{v\left[\mathcal{V}^{\prime}-(b+s) \mathcal{V}^{\prime \prime}\right] \Phi^{\prime} \mu^{2}-\left(\mathcal{F}_{k}-\delta\right) n^{2} \mathcal{V}^{\prime} \mathcal{V}^{\prime \prime} \mu} .
\end{aligned}
$$

\footnotetext{
${ }^{26}$ The matrix algebra manipulations in this appendix were conveniently performed using the Maple engine incorporated in Scientific Word.
} 
The implicit function theorem implies that the condition $\left(\mu_{t}=\mu_{t+1}\right)$ defines a continuous mapping of $\mu$ onto $a$. Similarly, the condition $\left(a_{t}=a_{t+1}\right)$ defines a continuous mapping of $a$ onto $\mu$.

Comparing (74) with (75), we obtain that for as long as $\mathcal{F}_{k}>\delta$ the following inequalities hold:

$$
\left(\frac{d a}{d \mu}\right)_{\mu_{t}=\mu_{t+1}}>-\frac{v}{\mu}>\left(\frac{d a}{d \mu}\right)_{a_{t}=a_{t+1}} .
$$

Since $\mu_{t}=\mu_{t+1}$ implies $\mathcal{F}_{k}>\delta$, the line $\mu_{t}=\mu_{t+1}$ is steeper than the line $a_{t}=a_{t+1}$ at all points the two lines cross. This property and the continuity of the two stationary lines imply that there is at most one steady state.

The exitence of a steady state is proved by construction as follows. The Inada conditions assumed on $\mathcal{F}$ imply that there is a unique value of the ratio $(k / n)$ to be denoted $(k / n)^{*}$ consistent with $\mu_{t}=\mu_{t+1}$. Therefore, there is a unique value of $\Phi$, to be denoted $\Phi^{*}$. Also, there is a unique value for the expression $\mathcal{F}\left(\left[(k / n)^{*}, 1\right]-\delta(k / n)^{*}\right.$ to be denoted $f^{*}$. The Euler condition and the definition of $(k / n)^{*}$ imply $f^{*}>0$. The stationary condition (39) and the unique value of $(k / n)^{*}$ consistent with (38) imply

$$
a=k+f^{*} n .
$$

Comparing (65) and (77) and using (68), we obtain

$$
n=\frac{\alpha_{1} \Phi^{*}}{f^{*}} \frac{1}{\mathcal{V}^{\prime}(l)}
$$

Combining (66), (67), and (78), we obtain the following equation with only one unkwon:

$$
(1-l) \mathcal{V}^{\prime}(l)=\frac{\alpha_{1} \Phi^{*}}{f^{*}}+\alpha_{2} \alpha_{3}
$$

This equation determines the value of $l$ in a steady state. The right-hand-side of this equation is a positive number. The left-hand-side of this equation is an donward sloping function going from $\infty$ when $l=0$ to 0 when $l=1$. Therefore, there is a unique solution $l^{*}$ to this equation. Denoting with an asterisk steady state values, $\mu^{*}$ and $n^{*}$ are obtained substituting $(k / n)^{*}$ and $l^{*}$ in (30) and (78). The value $k^{*}$ is obtained dividing $(k / n)^{*}$ by $n^{*}$. Finally, the value $a^{*}$ is obtained from $(77)$.

Let $\mathcal{G}: \Re^{2} \rightarrow R$ and $\mathcal{J}: \Re^{2} \rightarrow R$ be defined as $\mathcal{G}\left(\mu_{t}, a_{t}\right) \equiv \mu_{t}\left[1-\delta+\mathcal{F}_{k}\left(k_{t}, n_{t}\right)\right]^{-1} \beta^{-1}$ and $\mathcal{J}\left(\mu_{t}, a_{t}\right) \equiv y_{t}+k_{t}(1-\delta)$. In abbreviated form, the dynamic system (33) and (34) is:

$$
\begin{gathered}
\mu_{t+1}=\mathcal{G}\left(\mu_{t}, a_{t}\right), \text { and } \\
a_{t+1}=\mathcal{J}\left(\mu_{t}, a_{t}\right) .
\end{gathered}
$$


The dynamics around a neighborhood of the steady state is characterized by the eigenvalues of the matrix:

$$
A_{2}=\left[\begin{array}{cc}
\mathcal{G}_{\mu} & \mathcal{G}_{a} \\
\mathcal{J}_{\mu} & \mathcal{J}_{a}
\end{array}\right]
$$

The phase diagram in Figure 1 implies that one the these eigenvalues is greater than one and the other, corresponding to the stable arm, is smaller than one. Because the dynamic system is in discrete time, for monotonic convergence around a steady state the eigenvalue corresponding with the stable arm must be positive. Equivalently, the following determinant must be positive:

$$
\operatorname{det}\left(A_{2}\right)=\operatorname{det}\left[\begin{array}{cc}
1-\frac{\mu \mathcal{F}_{k k}}{\left(1-\delta+\mathcal{F}_{k}\right)} \frac{d\left(\frac{k}{n}\right)}{d \mu} & -\frac{\mu \mathcal{F}_{k k}}{\left(1-\delta+\mathcal{F}_{k}\right)} \frac{d\left(\frac{k}{n}\right)}{d a} \\
\frac{d([k(1-\delta)+y]}{d \mu} & \frac{d([k(1-\delta)+y]}{d a}
\end{array}\right]
$$

In general, I could not find a way of signing this determinant, but when $\mathcal{V}^{\prime \prime}=0$ it simplifies to

$$
\operatorname{det}\left(A_{2}\right)=\left(1-\delta+\mathcal{F}_{k}\right)>0
$$

Therefore, local convergence to the steady state is monotonic at least when $\mathcal{V}^{\prime \prime}=0$.

\section{Comparative analysis across steady states}

In abbreviated form, the steady state values of $b, s, n, l, k$, and $\mu$ are characterized by the following system of equations:

$$
\begin{aligned}
f\left(\frac{k}{n}\right) n \mu-\alpha_{1} & =0, \\
1-b-s-n-l & =0, \\
\mathcal{V}^{\prime}(l) s-\alpha_{3} & =0, \\
\Phi\left(\frac{k}{n}\right) \mu-\mathcal{V}^{\prime}(l) & =0, \\
s-\varphi(R) b & =0, \text { and } \\
\mathcal{F}_{k}\left(\frac{k}{n}\right)-\delta+1-\beta^{-1} & =0 .
\end{aligned}
$$

This system is obtained combining (8), (11), (27), (29), (30), (32), (38), and (39), where $\alpha_{1}$ and $\alpha_{3}$ are constants, $f(k / n)=[\mathcal{F}(k / n, 1)-\delta], \Phi(k / n)=\mathcal{F}_{n} /\left(1-\delta+\mathcal{F}_{k}\right)$, and $\varphi(R)$ is defined in (28). These definitions imply $\Phi^{\prime}>0$ and $\varphi^{\prime}>0$. Equation (90) implies that $k / n$, and hence $\Phi$ and $f$ can be treated as constants in comparisons across steady states. Differentiating equations (85) to (89) from the previous system, we obtain ${ }^{27}$ :

\footnotetext{
${ }^{27}$ These derivatives have been simplified using (85) to (90).
} 


$$
\left[\begin{array}{ccccc}
f n & 0 & 0 & 0 & f \mu \\
0 & -1 & -1 & -1 & -1 \\
0 & 0 & \mathcal{V}^{\prime} & s \mathcal{V}^{\prime \prime} & 0 \\
\mathcal{V}^{\prime} \mu^{-1} & 0 & 0 & -\mathcal{V}^{\prime \prime} & 0 \\
0 & -\varphi & 1 & 0 & 0
\end{array}\right]\left[\begin{array}{c}
d \mu \\
d b \\
d s \\
d l \\
d n
\end{array}\right]=\left[\begin{array}{c}
0 \\
0 \\
0 \\
0 \\
b \varphi^{\prime}
\end{array}\right] d R .
$$

Using standard linear algebra, we solve the previous equation to obtain:

$$
\left[\begin{array}{c}
d \mu \\
d b \\
d s \\
d l \\
d n
\end{array}\right]=\left[\begin{array}{c}
\mu \mathcal{V}^{\prime \prime} \\
-\mathcal{V}^{\prime}+(n+s) \mathcal{V}^{\prime \prime} \\
-s \mathcal{V}^{\prime \prime} \\
\mathcal{V}^{\prime} \\
-n \mathcal{V}^{\prime \prime}
\end{array}\right] A_{3} \frac{\varphi^{\prime}}{\varphi} d R
$$

where $A_{3}=b\left[\mathcal{V}^{\prime}-(1-l) \mathcal{V}^{\prime \prime}\right]^{-1}$. Total differentiation of the utility of a household (2) yields:

$$
\begin{aligned}
d W= & \frac{1}{q} d q+\frac{1}{1-\sigma} \frac{b+s}{b s}\left(\left(\frac{s}{b+s}\right)^{2} d b+\left(\frac{b}{b+s}\right)^{2} d s\right)+\mathcal{V}^{\prime} d l= \\
& \frac{\left.\mathcal{V}^{\prime \prime}(\sigma \varphi(1-l)-b-s)\right)+\mathcal{V}^{\prime}\left[\mathcal{V}^{\prime}(b+s)(1-\sigma)-\sigma \varphi\right]}{(1-\sigma)(1+\varphi)\left[\mathcal{V}^{\prime}-(1-l) \mathcal{V}^{\prime \prime}\right]} \frac{\varphi^{\prime}}{\varphi} d R .
\end{aligned}
$$

Using (29) and $s=\varphi b$, we find

$$
\mathcal{V}^{\prime}(b+s)(1-\sigma)-\varphi \sigma=\sigma \frac{2\left(1+\varphi-\varphi^{2}\right)-\sigma \varphi^{2}}{(2+\sigma) \varphi}<0 \text { for } \varphi \geq 2
$$

Comparison between an optimal and an equilibrium allocation with $R=0$ :

Equations (60) and (62) follow from a comparison of (24) and (28) with (57) and (58). In both steady states the net marginal product of capital is equal to the subjective discount rate, so (59) follows. Combining (29), (30), (31), and (62), we obtain:

$$
\frac{\sigma^{2}}{1-\sigma^{2}} \frac{1}{3} q=\frac{\mathcal{F}_{n}(k, n)}{1-\delta+\mathcal{F}_{k}(k, n)}
$$

Combining (51), (53), (55), (56), (57), and (62), we obtain:

$$
\frac{\sigma^{2}}{1-\sigma^{2}} \frac{1}{2} q^{*}=\frac{\mathcal{F}_{n}\left(k^{*}, n^{*}\right)}{1-\delta+\mathcal{F}_{k}\left(k^{*}, n^{*}\right)} .
$$

The right-hand-side of (95) and (96) are identical because of (59), so (63) follows. Condition (39) applies to both steady states. Using constant returns and (62), this condition implies:

$$
\left[\mathcal{F}\left(\frac{k}{n}, 1\right)-\delta \frac{k}{n}\right] n=\frac{1}{3} x q s, \text { and }
$$




$$
\left[\mathcal{F}\left(\frac{k^{*}}{n^{*}}, 1\right)-\delta \frac{k^{*}}{n^{*}}\right] n^{*}=\frac{1}{2} x^{*} q^{*} s^{*}
$$

These expressions together with (59), (60), and (63) imply (61). Combining (5), (51), (53), (57), and (62), we obtain:

$$
\mathcal{V}^{\prime}\left(l^{*}\right) s^{*}=\frac{\sigma}{1-\sigma} \frac{1}{2} .
$$

Combining (29) and (99), together with the logarithmic form of $\mathcal{V}$, we obtain (64).

\section{Proof of Proposition 4}

The random matching functions are:

$$
\begin{aligned}
& \mathcal{B}\left(b_{t}, B_{t}, \overline{B_{t}}, S_{t}\right)=b_{t} \frac{S_{t}}{B_{t}+\overline{B_{t}}+S_{t}}, \quad \mathcal{S}\left(s_{t}, B_{t}, \overline{B_{t}}, S_{t}\right)=s_{t} \frac{B_{t}}{B_{t}+\overline{B_{t}}+S_{t}}, \\
& \overline{\mathcal{B}}\left(\overline{b_{t}}, B_{t}, \overline{B_{t}}, S_{t}\right)=\overline{b_{t}} \frac{S_{t}}{B_{t}+\overline{B_{t}}+S_{t}}, \quad \overline{\mathcal{S}}\left(s_{t}, B_{t}, \overline{B_{t}}, S_{t}\right)=s_{t} \frac{\overline{B_{t}}}{B_{t}+\overline{B_{t}}+S_{t}} .
\end{aligned}
$$

Consumption is:

$$
c_{t}=\left[Q_{t}^{1-\sigma} \mathcal{B}\left(b_{t}, B_{t}, \bar{B}_{t}, S_{t}\right)\left(x_{t}-\frac{x_{t}^{2}}{2}\right)+\bar{Q}_{t}^{1-\sigma} \overline{\mathcal{B}}\left(\bar{b}_{t}, B_{t}, \bar{B}_{t}, S_{t}\right)\left(\bar{x}_{t}-\frac{\bar{x}_{t}^{2}}{2}\right)\right]^{\frac{1}{1-\sigma}}
$$

The labor resource constraint, the money budget constraint, and the constraint on the maximum number of buyers carrying money are the same as in Section 2. The stock of goods at the beginning of period $t$ is:

$$
\begin{aligned}
a_{t}= & y_{t}+k_{t-1}(1-\delta)+ \\
& {\left[\bar{b}_{t-1} \bar{Z}_{t-1}+\bar{z}_{t-1} \overline{\mathcal{X}}\left(\bar{q}_{t-1}, \bar{z}_{t-1}, t-1\right)-\bar{Z}_{t-1} \bar{x}_{t-1} \overline{\mathcal{S}}\left(\bar{b}_{t-1}, B_{t-1}, \bar{B}_{t-1}, S_{t-1}\right)\right]\left(1-\delta_{m}\right) . }
\end{aligned}
$$

The split of the initial goods must obey:

$$
a_{t}-v_{t}-k_{t}-\bar{b}_{t} \bar{Z}_{t}=0
$$

where

$$
v_{t}=q_{t} \mathcal{X}\left(q_{t}, z_{t}, t\right) \mathcal{S}\left(s_{t}, B_{t}, \bar{B}_{t}, S_{t}\right)+\bar{q}_{t} \overline{\mathcal{X}}\left(\bar{q}_{t}, \bar{z}_{t}, t\right) \overline{\mathcal{S}}\left(s_{t}, B_{t}, \bar{B}_{t}, S_{t}\right)
$$

The first order conditions (13) to (19) remain the same except for the obvious change in the arguments of the matching functions. In addition, we have the following first order conditions obtained from the optimal choice of $\bar{x}_{t}, \bar{q}_{t}, \bar{z}_{t}$ and $\bar{b}_{t}$, :

$$
\begin{gathered}
\mathcal{U}^{\prime}\left(c_{t}\right) c^{\sigma} \frac{\bar{Q}_{t}^{1-\sigma}}{1-\sigma}\left(1-\bar{x}_{t}\right)=\mu_{t+1} \beta\left(1-\delta_{m}\right) \bar{Z}_{t} \\
\mu_{t}\left[\overline{\mathcal{X}}\left(\bar{q}_{t}, \bar{z}_{t}, t\right)+\bar{q}_{t} \overline{\mathcal{X}}_{\bar{q}}\left(\bar{q}_{t}, \bar{z}_{t}, t\right)\right]=\mu_{t+1} \beta\left(1-\delta_{m}\right) \bar{z}_{t} \overline{\mathcal{X}}_{\bar{q}}\left(\bar{q}_{t}, \bar{z}_{t}, t\right)
\end{gathered}
$$




$$
\begin{gathered}
\mu_{t} \bar{q}_{t} \overline{\mathcal{X}}_{\bar{z}}\left(\bar{q}_{t}, \bar{z}_{t}, t\right)=\mu_{t+1} \beta\left(1-\delta_{m}\right)\left[\overline{\mathcal{X}}\left(\bar{q}_{t}, \bar{z}_{t}, t\right)+\bar{z}_{t} \overline{\mathcal{X}}_{\bar{z}}\left(\bar{q}_{t}, \bar{z}_{t}, t\right)\right] \\
\mathcal{U}^{\prime}\left(c_{t}\right) c^{\sigma} \frac{\bar{Q}_{t}^{1-\sigma}}{1-\sigma}\left(\bar{x}_{t}-\frac{\bar{x}_{t}^{2}}{2}\right) \overline{\mathcal{B}}_{1}\left(\bar{b}_{t}, B_{t}, \bar{B}_{t}, S_{t}\right) \leq \\
\begin{array}{r}
\mu_{t+1} \beta\left(1-\delta_{m}\right) \bar{Z}_{t} \bar{x}_{t} \overline{\mathcal{B}}_{1}\left(\bar{b}_{t}, B_{t}, \bar{B}_{t}, S_{t}\right) \\
+\mu_{t} \bar{Z}_{t}-\mu_{t+1} \beta\left(1-\delta_{m}\right) \bar{Z}_{t}+\mathcal{V}^{\prime}\left(l_{t}\right)
\end{array}
\end{gathered}
$$

The definition of symmetric equilibrium is analogous to the one in Section 2. The same steps as those in Section 2 yield the markup equation (25) and the following equations:

$$
\begin{gathered}
x_{t}=\bar{x}_{t}=\frac{\sigma}{1+\sigma} \\
\mu_{t+1} \beta\left(1-\delta_{m}\right) \bar{z}_{t}=(1-\sigma)^{-1} \mu_{t} \bar{q}_{t} .
\end{gathered}
$$

These two equations together with (13) and (106), and symmetry implies:

$$
\bar{q}_{t}=q_{t}
$$

Using the matching function definitions (100) and (101), and the relations (19), (26), and (110) to (112), the first order conditions for the optimal choice of $b_{t}$ and $\bar{b}_{t}$ in a symmetric equilibrium are transformed into:

$$
\begin{gathered}
\frac{\sigma^{2}}{2(1+\sigma)} \frac{s_{t}}{b_{t}+\bar{b}_{t}+s_{t}}-\frac{V^{\prime}\left(l_{t}\right)}{\mu_{t} q_{t}}(1-\sigma) \leq\left[1-\delta+\mathcal{F}_{k}\left(k_{t-1}, n_{t-1}\right)\right]\left(1+\pi_{t}\right)-1=R_{t}, \\
\frac{\sigma^{2}}{2(1+\sigma)} \frac{s_{t}}{b_{t}+\bar{b}_{t}+s_{t} t}-\frac{V^{\prime}\left(l_{t}\right)}{\mu_{t} q_{t}}(1-\sigma) \leq \frac{1-\delta+\mathcal{F}_{k}\left(k_{t}, n_{t}\right)}{1-\delta_{m}}-1=\rho_{t} .
\end{gathered}
$$

Finally, the inequalities (113) and (114) imply the proposition. 
Table 1

\section{THE COST OF INFLATION WITH BILATERAL MATCHING}

Production function: $\mathcal{F}\left(k_{t}, e_{t} n_{t}\right)=k_{t}^{0.36}\left(e_{t} n_{t}\right)^{0.64}$

Depreciation rate: $0.1 / T$

Commercial markup: $(1-\sigma)^{-1}=1.4$

One Period Utility: $\ln \left(c_{t}\right)+0.81 \ln \left(l_{t}\right)$

Rate of growth of $e_{t}: g=0.018 / T$

Real interest rate: $r=0.04 / T$

Discount factor: $\beta=(1+g) /(1+r)$

Opportunity cost of holding money: $R=r+(\pi / T)$

Number of periods per year: $T=76$

\begin{tabular}{lccccccc} 
& \multicolumn{7}{c}{ Annual Inflation Rate $(\pi)$} \\
\cline { 2 - 8 } & -0.04 & 0 & 0.029 & 0.05 & 0.10 & 0.20 & 0.50 \\
\hline Producers $(n)$ & 0.376 & 0.376 & 0.377 & 0.377 & 0.378 & 0.380 & 0.385 \\
Home Workers $(l)$ & 0.379 & 0.380 & 0.380 & 0.380 & 0.381 & 0.383 & 0.388 \\
Sellers $(s)$ & 0.164 & 0.164 & 0.164 & 0.164 & 0.165 & 0.166 & 0.168 \\
Buyers $(b)$ & 0.082 & 0.080 & 0.079 & 0.077 & 0.075 & 0.071 & 0.059 \\
Individuals at Home $(b+l)$ & 0.461 & 0.460 & 0.459 & 0.457 & 0.456 & 0.454 & 0.447 \\
Annual Velocity & 11.26 & 11.34 & 11.42 & 11.47 & 11.59 & 11.82 & 12.52 \\
Welfare Cost of Inflation & -0.254 & 0 & 0.192 & 0.336 & 0.693 & 1.490 & 4.588 \\
Net Benefit National Currency & 0.449 & 0.193 & 0 & -0.144 & -0.504 & -1.304 & -4.412
\end{tabular}

Note: The welfare cost of inflation is the compensating variation on $a_{t}$ when inflation drops from $\pi$ to 0 . The net benefit of national currency is the welfare cost of inflation minus the real value of the national currency. This benefit is based on the assumption that the economy would achieve price stability with either gold or foreign dollars. The welfare cost of inflation and the net benefit of national currency are in percent of global wealth (present value of lifetime market consumption expenditures). These costs and 
benefits include those during the transitions across steady states. The other variables in the table are steady state values. 
Table 2

\section{THE COST OF INFLATION WITH A CASH-IN-ADVANCE CONSTRAINT}

Production function: $\mathcal{F}\left(k_{t}, e_{t} n_{t}\right)=k_{t}^{0.36}\left(e_{t} n_{t}\right)^{0.64}$

Depreciation rate: $0.1 / T$

One Period Utility: $\ln \left(c_{t}\right)+0.78 \ln \left(l_{t}\right)$

Rate of growth of $e_{t}: g=0.018 / T$

Real interest rate: $r=0.04 / T$

Discount factor: $\beta=(1+g) /(1+r)$

Opportunity cost of holding money: $R=r+(\pi / T)$

Number of periods per year: $T=11.34$

Annual Inflation Rate $(\pi)$

\begin{tabular}{|c|c|c|c|c|c|c|c|}
\hline & \\
\hline & -0.04 & 0 & 0.05 & 0.10 & 0.20 & 0.50 & 1.12 \\
\hline Producers $(n)$ & 0.540 & 0.540 & 0.538 & 0.537 & 0.535 & 0.529 & 0.517 \\
\hline Individuals at Home $(l)$ & 0.460 & 0.460 & 0.462 & 0.463 & 0.465 & 0.471 & 0.483 \\
\hline Annual Velocity & 11.34 & 11.34 & 11.34 & 11.34 & 11.34 & 11.34 & 11.34 \\
\hline Welfare Cost of Inflation & -0.0003 & 0 & 0.001 & 0.003 & 0.009 & 0.046 & 0.193 \\
\hline Net Benefit National Currency & 0.194 & 0.193 & 0.192 & 0.190 & 0.194 & 0.147 & 0 \\
\hline
\end{tabular}

Note: The weight parameter in the one period utility function and $T$ has been adjusted so the fraction of individuals at home and the annual velocity is the same as those in Table 1 when $\pi=0$. See the note in Table 1 for definitions. 
Table 3

SENSITIVITY TO ALTERNATIVE PARAMETERS OF THE COST OF A 10 PERCENT RATE OF INFLATION WITH BILATERAL MATCHING

Production function: $\mathcal{F}\left(k_{t}, e_{t} n_{t}\right)=k_{t}^{0.36}\left(e_{t} n_{t}\right)^{0.64}$

Depreciation rate: $0.1 / T$

One Period Utility: $\ln \left(c_{t}\right)+\theta \ln \left(l_{t}\right)$

Rate of growth of $e_{t}: g=0.018 / T$

Real interest rate: $r$

Discount factor: $\beta=(1+g) /(1+r)$

Opportunity cost of holding money: $R=r+(\pi / T)$

\begin{tabular}{lcccccc} 
& \multicolumn{7}{c}{ Alternative Parameter Sets } \\
\cline { 2 - 7 } & $(1)$ & $(2)$ & $(3)$ & $(4)$ & $(5)$ & $(6)$ \\
Annual Interest Rate $(r T)$ & 0.02 & 0.065 & 0.04 & 0.04 & 0.04 & 0.04 \\
Commercial markup $(1-\sigma)^{-1}$ & 1.4 & 1.4 & 1.8 & 1.2 & 1.4 & 1.4 \\
Number of Periods per Year $(T)$ & 76 & 76 & 55 & 117 & 40 & 152 \\
Weight of Home Services $(\theta)$ & 0.86 & 0.77 & 0.88 & 0.79 & 0.81 & 0.81 \\
\hline Producers $(n)$ & 0.386 & 0.371 & 0.290 & 0.446 & 0.379 & 0.377 \\
Home workers $(l)$ & 0.385 & 0.378 & 0.338 & 0.415 & 0.385 & 0.380 \\
Sellers $(s)$ & 0.156 & 0.172 & 0.253 & 0.097 & 0.165 & 0.164 \\
Buyers $(b)$ & 0.072 & 0.079 & 0.119 & 0.042 & 0.070 & 0.079 \\
Individuals at Home $(b+l)$ & 0.457 & 0.457 & 0.457 & 0.457 & 0.455 & 0.459 \\
Annual Velocity & 11.57 & 11.57 & 11.49 & 11.74 & 6.30 & 22.85 \\
Welfare Cost of Inflation & 0.663 & 0.728 & 0.955 & 0.607 & 1.484 & 0.325 \\
Net Benefit National Currency & -0.645 & -0.325 & -0.764 & -0.419 & -1.133 & -0.229
\end{tabular}

Note: The parameters $T$ and $\theta$ are calibrated so at zero inflation individuals at home and annual velocity match the following values: individuals at home 0.46 in all columns, annual velocity 11.34 in columns (1) to (4), 6.07 in column (5), and 22.68 in column (6). See the note in Table 1 for definitions. 
Figure 1

TRANSITIONAL DYNAMICS

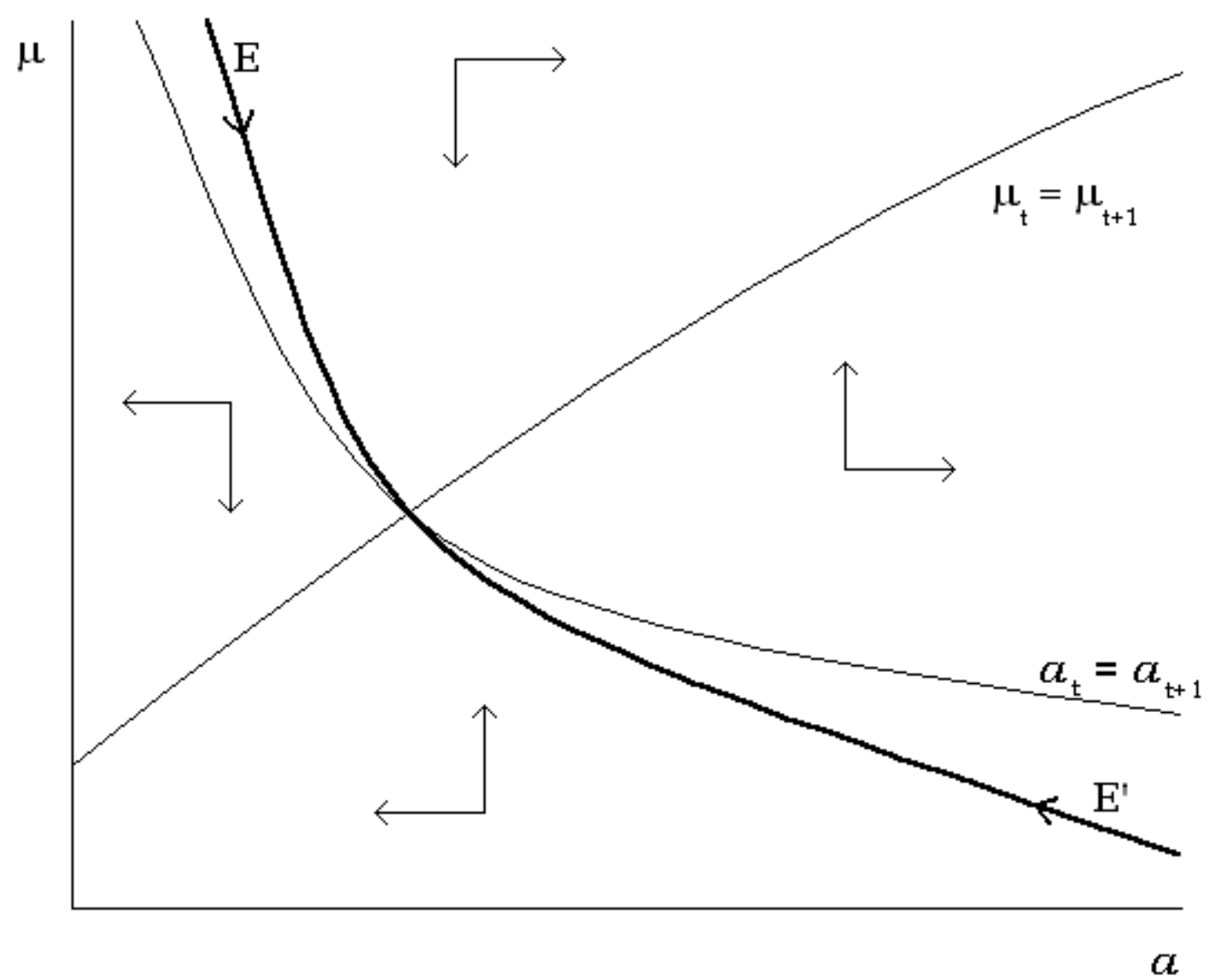

